Linköping Studies in Science and Technology. Dissertation No. 1871

\title{
Toward the Optimization of Low-temperature Solution-based Synthesis of ZnO Nanostructures for Device Applications
}

\author{
Hatim Alnoor
}

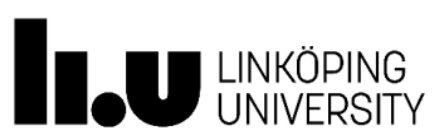

Physical Electronics and Nanotechnology Group

Department of Science and Technology (ITN)

Linköping University, SE-601 74 Norrköping, Sweden

2017 Norrköping 
Cover Picture: The light emission from the fabricated heterojunction LEDs within the thesis.

Photo credit: Thor Balkhed, Linköping University.

During the course of research underlying this thesis, Hatim Alnoor was enrolled in Agora Materiae, a multidisciplinary doctoral program at Linköping University, Sweden.

(C) Hatim Alnoor, 2017

hatim.alnoor@liu.se

hatimkafi79@gmail.com

Printed by LiU-Tryck, Linköping, Sweden, 2017

ISBN: 978-91-7685-481-5

ISSN 0345-7524

Electronic publication: www.ep.liu.se 
Dedicated to

My family 



\begin{abstract}
One-dimensional (1D) nanostructures (NSs) of Zinc Oxide ( $\mathrm{ZnO})$ such as nanorods (NRs) have recently attracted considerable research attention due to their potential for the development of optoelectronic devices such as ultraviolet (UV) photodetectors and light-emitting diodes (LEDs). The potential of $\mathrm{ZnO} \mathrm{NRs}$ in all these applications, however, would require synthesis of high crystal quality $\mathrm{ZnO}$ NRs with precise control over the optical and electronic properties. It is known that the optical and electronic properties of $\mathrm{ZnO}$ NRs are mostly influenced by the presence of native (intrinsic) and impurities (extrinsic) defects. Therefore, understanding the nature of these intrinsic and extrinsic defects and their spatial distribution is critical for optimizing the optical and electronic properties of $\mathrm{ZnO}$ NRs. However, identifying the origin of such defects is a complicated matter, especially for NSs, where the information on anisotropy is usually lost due to the lack of coherent orientation. To investigate the origin of these defects in more details and to fulfill all the advantages of $\mathrm{ZnO}$ NRs in device applications several synthesis techniques have been utilized. Among them, the low-temperature solution-based methods, which are regarded as promising due to many advantages. It is low-cost and offers the possibility of large-scale production and tuning the properties of the final product through the synthesis parameters. However, synthesizing reproducible $\mathrm{ZnO}$ NRs with optimized morphology, orientation, electronic and optical properties by the low-temperature solution-based methods remains a challenge.
\end{abstract}

Thus, the aim of this thesis is towards the optimization of the lowtemperature solution-based synthesis of $\mathrm{ZnO}$ NRs for device applications. In this connection, we first started with investigating the effect of the 
precursor solution stirring durations on the deep level defects concentration and their spatial distribution along the $\mathrm{ZnO}$ NRs. Then, by choosing the optimal stirring time, we studied the influence of $\mathrm{ZnO}$ seeding layer precursor's types and its molar ratios on the density of interface defects. The findings of these investigations were used to demonstrate $\mathrm{ZnO}$ NRsbased heterojunction LEDs. The ability to tune the point defects along the NRs enabled us further to incorporate cobalt (Co) ions into the $\mathrm{ZnO}$ NRs crystal lattice, where these ions could occupy the vacancies or interstitial defects through substitutional or interstitial doping. Following this, high crystal quality vertically well-oriented $\mathrm{ZnO}$ NRs have been demonstrated by incorporating a small amount of $\mathrm{Co}$ into the $\mathrm{ZnO}$ crystal lattice. Finally, the influence of Co ions incorporation on the reduction of core-defects (CDs) in $\mathrm{ZnO} \mathrm{NRs}$ was systematically examined using electron paramagnetic resonance (EPR). 


\section{Acknowledgment}

First, I must acknowledge and thank The Almighty Allah for blessing, protection, and for guiding me through this journey and all my life. This thesis would not have been feasible without the help, support, and encouragement of many people to whom I am sincerely thankful.

I would like to express my deepest gratitude to my supervisor, associate Prof. Omer Nour who believed in my knowledge during the first time I met him and gave me this great opportunity to pursue my Ph.D. degree under his supervision in the Physical Electronics and Nanotechnology group. Thanks Omer for endless guidance, support, motivation, encouragement, and patient help during the course of my Ph.D. Your guidance, encouragement and enough freedom during my research encouraged me to grow my knowledge and to become an independent researcher. Also, I would like to thank, Omer and his family for the generous hospitality at their home when I first arrived at Norrköping.

I express my profound gratitude to my co-supervisor, Prof. Magnus Willander, for his support, encouragement, valuable discussions, positive comments, and suggestions during my Ph.D. research course.

I am extremely grateful to all my co-authors Galia Pozina, Adrien Savoyant, Volodymyr Khranovskyy, and Xianjie Liu for collaboration, discussions, sharing your knowledge, suggestions, and for contribution in the correction of some parts in the thesis manuscript.

Fredrik Eriksson thanks for your great help with XRD measurements and for correcting the XRD part in this thesis.

Chan Oeurn Chey and Zia Ullah many thanks to all of you for teaching me how to work in the lab and for the great help in some measurements. 
Ildiko Farkas thanks for helping with cutting samples.

Special thanks to Ann-Christin Norén for kind help and advice and taking care of all our research administration work.

Anna Malmström, Lars Gustavsson, and Putte Eriksson for keeping the lab running in good condition.

My thanks to Thor Balkhed for crediting the photo of the cover picture and for help in the design of the cover picture.

The past and present Physical Electronics and Nanotechnology group members for support, collaboration, and encouragement.

The past and present members of the research school Agora Materiae, and special thanks to Prof. Per-Olof Holtz for organizing interesting seminars, summer conference, study visits, and annual follow-up meetings. Also, special thanks for Karina Malmström for taking care of all Agora Materiae administration work.

I am very grateful to The National Center for Research (Sudan), National Council for Training (Sudan), Ministry of Higher Education, and Scientific Research (Sudan) and Linköping University for their financial support during my Ph.D. study.

I would like to thank all friends and colleagues whom I met during my $\mathrm{Ph} . \mathrm{D}$. for their support, encouragement and sharing a good time.

My uncle Eltahir, cousin Hamad and friends Ali and Adel for their support for this scholarship to be possible.

I would like to share my depths gratitude with my family, in particular, my parents, brothers and my sister for support, encouragement, prayers and for 
wishing me all the best during my Ph.D. study and in my life. Also, I express my appreciation to all the members of my family-in-law for their support and engagement.

My lovely wife Tyseer words cannot express my gratitude to you. Thanks for love, joyousness, patience, support, and being in my life. 


\section{List of the papers included in this thesis}

1. Effect of precursor solution stirring on deep level defects concentration and spatial distribution in low temperature aqueous chemical synthesis of zinc oxide nanorods

Hatim Alnoor, Chan Oeurn Chey, Galia Pozina, Xianjie Liu, Volodymyr Khranovskyy, Magnus Willander, and Omer Nur. AIP Advances 5, 087180 (2015).

Contribution: I performed all the experimental work except the PL and XPS, and I wrote the first draft of the manuscript.

2. Influence of $\mathrm{ZnO}$ seed layer precursor molar ratio on the density of interface defects in low temperature aqueous chemically synthesized $\mathrm{ZnO}$ nanorods/GaN light-emitting diodes

Hatim Alnoor, Galia Pozina, Volodymyr Khranovskyy, Xianjie Liu, Donata Iandolo, Magnus Willander, and Omer Nur.

J. Appl. Phys. 119, 165702 (2016).

Contribution: I performed all the experimental work except the XPS, PL, and AFM. I wrote the first version of the manuscript.

3. Seed layer synthesis effect on the concentration of interface defects and emission spectra of $\mathrm{ZnO}$ nanorods/p-GaN light-emitting diode

Hatim Alnoor, Galia Pozina, Magnus Willander, and Omer Nur. Phys. Status Solidi A 214, 1600333 (2017).

Contribution: I performed all the experimental work and wrote the first version of the manuscript.

4. EPR investigation of pure and Co-doped $\mathrm{ZnO}$ oriented nanocrystals A. Savoyant, H. Alnoor, S. Bertaina, O. Nur, and M. Willander. Nanotechnology 28, 035705 (2017). 
Contribution: I performed the growth of the samples, did the SEM measurements, and contributed to the writing of the first version of the manuscript.

5. An effective low-temperature solution synthesis of Co-doped [0001]oriented $\mathrm{ZnO}$ nanorods

Hatim Alnoor, Adrien Savoyant, Xianjie Liu, Galia Pozina, Magnus Willander, and Omer Nur.

J. Appl. Phys. 121, 215102 (2017).

Contribution: I performed all the experimental work except the EPR, and XPS measurements. I wrote the first version of the manuscript.

6. Core-defect reduction in $\mathrm{ZnO}$ nanorods by cobalt incorporation

A. Savoyant, H. Alnoor, O. Pilone, O. Nur, and M. Willander. Nanotechnology 28, 285705 (2017).

Contribution: I grew the samples and performed SEM measurements. Also, I contributed to the writing of part of the first version of the manuscript. 


\section{List of the papers not included in this thesis}

1. Fast synthesis, morphology transformation, structural and optical properties of $\mathrm{ZnO}$ nanorods grown by seed-free hydrothermal method Chan Oeurn Chey, Hatim Alnoor, Mazhar Ali Abbasi, Omer Nur, and Magnus Willander.

Phys. Status Solidi A 211, 2611 (2014).

2. Fast piezoresistive sensor and UV photodetector based on Mn-doped $\mathrm{ZnO}$ nanorods

Chan Oeurn Chey, Xianjie Liu, Hatim Alnoor, Omer Nur, and Magnus Willander.

Phys. Status Solidi RRL 9, 87 (2015).

3. Zinc Oxide Nanostructure-Modified Textile and Its Application to Biosensing, Photocatalysis, and as Antibacterial Material Amir Hatamie, Azam Khan, Mohsen Golabi, Anthony P. F. Turner, Valerio Beni, Wing Cheung Mak, Azar Sadollahkhani, Hatim Alnoor, Behrooz Zargar, Sumaira Bano, Omer Nur, and Magnus Willander. Langmuir 31, 10913 (2015).

\section{Conference papers:}

4. Zinc oxide nanostructures and its nano-compounds for efficient visible light photo-catalytic processes

Rania E. Adam, Hatim Alnoor, Sami Elhag, Omer Nur, and Magnus Willander.

Proc. SPIE 10105, Oxide-based Materials and Devices VIII, 101050X (February 24, 2017); doi:10.1117/12.2254872.

5. Comparison between different metal oxide nanostructures and nanocomposites for sensing, energy generation and energy harvesting 
Magnus Willander, Hatim Alnoor, Sami Elhag, Zafar Hussain Ibupoto, Eiman Satti Nour, and Omer Nur.

Proc. SPIE 9749, Oxide-based Materials and Devices VII, 97491L (27 February 2016); doi: 10.1117/12.2214513. 


\section{List of Abbreviations}

\begin{tabular}{|c|c|}
\hline NSs & Nanostructures \\
\hline $0 \mathrm{D}$ & Zero-dimensional \\
\hline $1 \mathrm{D}$ & One-dimensional \\
\hline NRs & Nanorods \\
\hline $2 \mathrm{D}$ & Two-dimensional \\
\hline $\mathrm{ZnO}$ & Zinc oxide \\
\hline UV & Ultraviolet \\
\hline LEDs & Light-emitting diodes \\
\hline $\mathrm{CB}$ & Conduction band \\
\hline VB & Valence band \\
\hline $\mathrm{Co}$ & Cobalt \\
\hline $\mathrm{CDs}$ & Core defects \\
\hline EPR & Electron paramagnetic resonance \\
\hline IEP & Isoelectric point \\
\hline NBE & Near-band-edge emission \\
\hline DLE & Deep level emission \\
\hline $\mathrm{V}_{\mathrm{O}}$ & Oxygen vacancy \\
\hline $\mathrm{V}_{\mathrm{Zn}}$ & Zinc vacancy \\
\hline $\mathrm{OH}$ & Hydroxyl ion \\
\hline
\end{tabular}




$\begin{array}{ll}\mathrm{O}_{\mathrm{i}} & \text { Oxygen interstitials } \\ \text { DMSs } & \text { Diluted magnetic semiconductors } \\ \text { TMs } & \text { Transition metals } \\ \text { RT-FM } & \text { Room-temperature ferromagnetism } \\ \text { HMTA } & \text { Hexamethylenetetramine } \\ \text { pH } & \text { Power of hydrogen, Potential hydrogen } \\ \text { NH } & \text { Ammonia } \\ \text { LT-ACS } & \text { Low-temperature aqueous synthesis } \\ \text { Ag } & \text { Silver } \\ \text { GaN } & \text { Gallium nitrate } \\ \text { DI-water } & \text { Deionized water } \\ \text { TF } & \text { Thin film } \\ \text { NPs } & \text { Nanoparticles } \\ \text { KOH } & \text { Potassium hydroxide } \\ \text { h } & \text { Hours }\end{array}$

$\begin{array}{ll}\text { AFM } & \text { Atomic force microscope } \\ \text { rpm } & \text { Round per minute } \\ \mathrm{Ni} & \text { Nickel } \\ \mathrm{Au} & \text { Gold } \\ \mathrm{RIE} & \text { Reactive ion etching } \\ \text { xii } & \end{array}$




$\begin{array}{ll}\text { FE-SEM } & \text { Field-emission-scanning electron microscopy } \\ \text { XRD } & \text { X-ray diffraction } \\ \text { XPS } & \text { X-ray photoelectron spectroscopy } \\ \text { UHV } & \text { Ultra-high vacuum } \\ \mathrm{CL} & \text { Cathodoluminescence } \\ \mathrm{I}-\mathrm{V} & \text { Current }- \text { Voltage } \\ \mathrm{SMUs} & \text { Sources-measurement units } \\ \mathrm{EL} & \text { Electroluminescence } \\ B & \text { Magnetic field } \\ \mathrm{h} v & \text { Photon energy } \\ \Delta \mathrm{E} & \text { Energy difference } \\ \mathrm{DL} & \text { Deep level } \\ \mathrm{Zn} \mathrm{n}_{\mathrm{i}} & \text { Zinc interstitial } \\ \mathrm{Fe} & \text { Iron } \\ \mathrm{Mn} & \text { Manganese } \\ \mathrm{Cu} & \text { Copper } \\ \mathrm{ODMR} & \text { Optically detected magnetic resonance } \\ \mathrm{B} & \end{array}$




\section{List of figures}

Figure 2.1: The unit cell of the $\mathrm{ZnO}$ hexagonal wurtzite-type structure ...6

Figure 2.2: Schematic illustration of the $\mathrm{CB}$ and $\mathrm{VB}$ structure of $\mathrm{ZnO}$ in the vicinity of the fundamental bandgap....................................

Figure 2.3: CL spectra of the $\mathrm{ZnO}$ synthesized on sapphire substrate using the low-temperature aqueous chemical synthesis at $90{ }^{\circ} \mathrm{C}$ from a growth solution of a $0.075 \mathrm{M}$ concentration of hexamethylenetetramine (HMTA) and zinc nitrate hexahydrate in a deionized (DI)-water.................. 8

Figure 2.4: (a) Electronic configuration of the $3 d^{7}$ of $\mathrm{Co}^{2+}$. (b) Level diagram of $\mathrm{Co}^{2+}\left(3 \mathrm{~d}^{7}\right)$ ion in $\mathrm{ZnO}$, under successive application of free ion, crystal field, spin orbital, Zeeman coupling, and hyperfine interaction. Red arrows depict allowed EPR transitions between non-degenerate hyperfine levels

Figure 3.1: Surface topography of the prepared seeds layer using zinc (II) acetate dihydrate and $\mathrm{KOH}$ with a molar ratio of (a) 1:1, (b) 1:3, and (c) 1:5 M. Scanned area: 2 × $2 \mu \mathrm{m}$

Figure 3.2: Top-view FE-SEM images of the synthesized pure NRs grown using $0.05 \mathrm{M}$ concentration of zinc nitrate and $0.075 \mathrm{M}$ concentration of HMTA at $80^{\circ} \mathrm{C}$ for $6 \mathrm{~h}$ .16

Figure 3.3: Top-view FE-SEM images of the synthesized Co-doped $\mathrm{ZnO}$ NRs grown using the first and second method for preparing the synthesis solution (a), and (b), respectively....

Figure 3.4: (a) Schematic diagram illustrating the fabricated heterojunction LEDs, (b) Cross-sectional SEM image of the synthesized nZnO NRs on the $\mathrm{p}-\mathrm{GaN}$ substrate, (c) Optical photograph of the fabricated 
heterojunction LEDs on p-GaN substrate, and (d) Optical photograph of the fabricated heterojunction LEDs under forwarding bias................18

Figure 4.1: Schematic illustration of $x$-ray diffraction.................21

Figure 4.2: Schematic illustration of the XPS experiment..............22

Figure 4.3: Schematic illustration of the signals from the sample under electron beam bombardment.........................................23

Figure 4.4: Optical photograph illustrating the light emission from the fabricated $\mathrm{ZnO} \mathrm{NRs} / \mathrm{GaN}$ heterojunction LED under forwarding bias....24

Figure 4.5: Schematic illustration of the EPR experiment..............25

Figure 4.6: Schematic illustrations of the NRs samples and definition of the angle $\theta$ between the magnetic field $\mathrm{B}$ and the c-axis of the NRs. The microwave magnetic field $\mathrm{B}_{l}$ is perpendicular to both $\mathrm{B}$ and $\mathrm{C}$ .26

Figure 5.1: Top-view FE-SEM images of the synthesized $\mathrm{ZnO}$ NRs prepared under stirring durations of (a) $1 \mathrm{~h}$, (b) $3 \mathrm{~h}$, (C) $5 \mathrm{~h}$, (c), and (d) 15

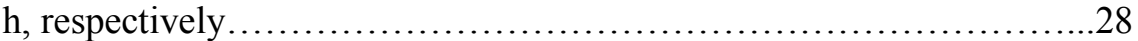

Figure 5.2: CL spectra of the individual NRs measured in cross-sectional view. The insets display the cross-sectional SEM images of the NRs with a sign of the spots where the CL spectra were recorded. For clarity, spectra have been offset in the vertical direction.............................30

Figure 5.3: CL spectra of $\mathrm{n}-\mathrm{ZnO} \mathrm{NRs} / \mathrm{p}-\mathrm{GaN}$ heterostructure recorded in cross-sectional view. The inset displays a typical cross-sectional SEM image of the n-ZnO NRs / p-GaN heterostructure with the sign of the point where the CL spectra were recorded.................................32 
Figure 5.4: EL spectra as a function of the forward bias voltage of device (a) 1, (b) 3, and (c) 5, respectively. The insets present the corresponding light emission images at $24 \mathrm{~V}$. (d) The integrated EL intensities of three devices as a function of the forward bias voltage.........................33

Figure 5.5: Integrated EL intensities of all the three devices as a function of the forward injection current. The solid lines represent the fitting results based on the power law $L=c I^{m}$

Figure 5.6: (a) $\mathrm{CL}$ spectra of the $\mathrm{n}-\mathrm{ZnO} \mathrm{NRs} / \mathrm{p}-\mathrm{GaN}$ heterostructures recorded in top-view mode. For clarity, all the spectra are normalized to the DL emission and shifted in vertical direction. (b) Cross-sectional view $\mathrm{CL}$ spectra of the $\mathrm{n}-\mathrm{ZnO} \mathrm{NRs} / \mathrm{p}-\mathrm{GaN}$ heterostructures interface. The insets display the synthesized $\mathrm{n}-\mathrm{ZnO} \mathrm{NRs} / \mathrm{p}-\mathrm{GaN}$ heterostructures in crosssectional view with a sign of the points where the CL spectra were measured 36

Figure 5.7: EL spectra of (a) ZK device, and (b) ZKH device, as a function of the bias voltage. The insets show the corresponding light emission images at $15 \mathrm{~V}$. (c) Gaussian decomposition of the dominant yellow emission of the ZKH device at a bias voltage of $15 \mathrm{~V}$. (d) EL spectra of the

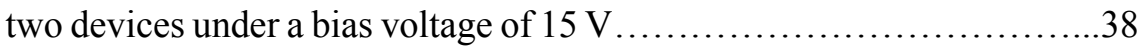

Figure 5.8: (a) The integrated EL intensities of the ZH and ZKH devices as a function of bias voltage. (b) Role of injection currents. The solid lines in Figure $\mathrm{b}$ illustrated the fitting results based on the power law $L=$

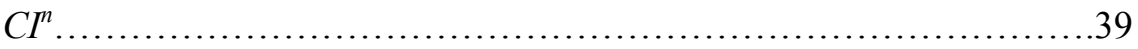

Figure 5.9: Top-view SEM images of the pure $\mathrm{ZnO}(\mathrm{S} 0)$ and 5\% Co-doped ZnO NRs (S2)

Figure 5.10: $X$-band EPR spectra (5 G modulation) at each step of the NRs synthesis. EPR of (a) The sample holder, (b) Sapphire substrate, (c) ZnO 
seed layer, (d) Pure ZnO NRs, and (e) 5\% of Co-doped ZnO NRs. No baseline subtraction or exponential smoothing.

Figure 5.11: Experimental $X$-band EPR spectra( $1 \mathrm{G}$ modulation) of sample S0 showing the $\mathrm{g} \sim 1.96$ signal for $\mathrm{B} / / \mathrm{z}\left(\theta=0^{\circ}\right)$ and $\mathrm{B} \perp \mathrm{z}\left(\theta=90^{\circ}\right)$, recorded at $\mathrm{T}=5 \mathrm{~K}$. The upper $\mathrm{x}$-axis gives the corresponding $\mathrm{g}$ factor value.

Figure 5.12: X-band EPR spectra of sample $S 2$ for parallel $\left(\theta=0^{\circ}\right)$ and perpendicular $\left(\theta=90^{\circ}\right)$ orientation of $\mathrm{B}$ field. The $\mathrm{CD}$ and sample holder $(\mathrm{SH})$ signals are shown. Gravity centers of the $\mathrm{Co}^{2+}$ signal for both orientations are shown

Figure 5.13: Top-view FE-SEM images of pure (M0) and Co-doped $\mathrm{ZnO}$ NRs synthesized using approaches M1 and M2, respectively....

Figure 5.14: XRD patterns of the synthesized pure (M0) and Co-doped ZnO NRs (M1and M2). The inset shows the normalized XRD data for the (002) peaks, indicating peak shifts

Figure 5.15: EPR spectra of Co-doped $\mathrm{ZnO}$ NRs (M1 and M2) for parallel $\left(\theta=0^{\circ}\right)$ and perpendicular $\left(\theta=90^{\circ}\right)$ orientation of magnetic field, recorded at $\mathrm{T}=5 \mathrm{~K}$. .46

Figure 5.16: $\mathrm{CL}$ spectra of the synthesized pure and Co-doped $\mathrm{ZnO}$ NRs synthesized utilizing different synthesis preparation approaches as shown. The inset demonstrates the red-shift in the UV peak. For clarity, the spectra are normalized to the NBE intensity....

Figure 5.17: Schematic diagram of the cross-sectional view of the synthesized pure and Co-doped $\mathrm{ZnO}$ NRs including $\mathrm{Zn}_{\mathrm{i}}^{+}$as core-defect and oxygen vacancies/interstitials as surface defects, respectively 48 
Figure 5.18: Evolution of the $C D$ signal with the cobalt concentration within the synthesis solution. The line of the $0 \%$ is divided by $100 \ldots \ldots . .50$

Figure 5.19: Anisotropy of the EPR CD signal in pure $(0 \% \mathrm{Co})$ and Codoped $(0.5 \%)$ samples, with respect to the magnetic field orientation, recorded at $\mathrm{T}=6 \mathrm{~K}$. Sapphire defect $(\mathrm{SD})$ signal present in the $0.5 \%$ sample is shown for the $\theta=0^{\circ}$ orientation..................................52 


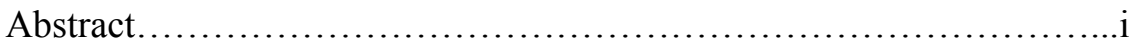

Acknowledgment...................................................ii

List of the papers included in the thesis..............................vii

List of the papers not included in the thesis............................ix

List of Abbreviations.................................................

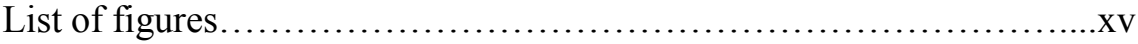

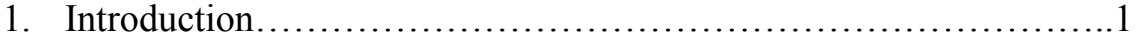

2. Material characteristics........................................ 5

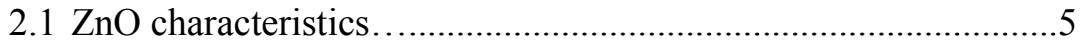

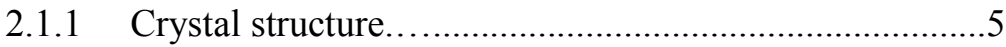

2.1.2 Electronic band structure.............................................6

2.1.3 Optical characteristics...................................................

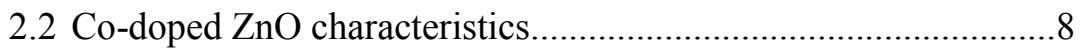

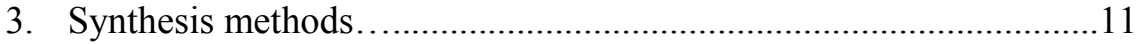

3.1 Hydrothermal synthesis methods.....................................11

3.2 $\mathrm{ZnO}$ seed layer preparation..........................................................13

3.3 Synthesis of $\mathrm{ZnO}$ NRs....................................14

3.4 Synthesis of Co-doped ZnO NRs...........................................15

3.5 N-ZnO NRs/p-GaN-based heterojunction LEDs..........................17

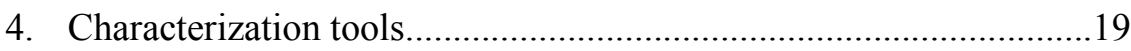

4.1 Scanning electron microscopy (SEM) ......................................19

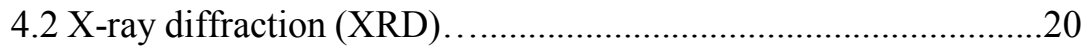

4.3 X-ray photoelectron spectroscopy (XPS).................................21

4.4 Photoluminescence (PL) spectroscopy.........................................22

4.5 Cathodoluminescence (CL) spectroscopy....................................22

4.6 Current-Voltage (I-V) measurements........................................23 
4.7 Electroluminescence (EL) measurements.....................24

4.8 Electron Paramagnetic Resonance (EPR) measurements..............24

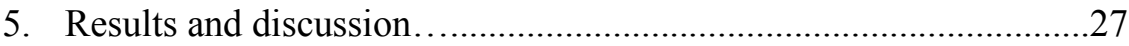

5.1 The effect of precursor (solutions) stirring durations on the optical

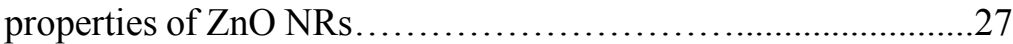

5.2 Influence of $\mathrm{ZnO}$ seed layer precursor molar ratios on the emission properties of n-ZnO NRs-based heterojunction LEDs............31

5.3 Influence of $\mathrm{ZnO}$ seed layer precursor types on the emission properties of $\mathrm{n}-\mathrm{ZnO}$ NRs-based heterojunction LEDs.............35

5.4 Synthesis of Co-doped $\mathrm{ZnO}$ oriented NRs..............................39

5.5 The influence of the synthesis solution mixing on Co-doped $\mathrm{ZnO}$ NRs.........................................................43

5.6 Core-defect reduction in ZnO NRs by Co incorporation...............49

6. Research summary and future work................................................53

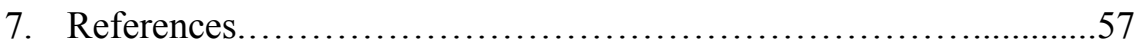




\section{Introduction}

The ability to synthesize materials at the nanoscale range not only offers a way for exploring their fascinating physical, chemical, mechanical and biological properties but also for their potential as building blocks for the development of a diverse range of emerging electronic devices and systems. ${ }^{[1-3]}$ Synthesis of such remarkable nanomaterials can be achieved by either assembling the atoms/molecules (bottom-up approach) or sculpting the bulk solids into small atomic level pieces (top-down approach) to obtain the desired nanostructures (NSs). Such NSs materials can typically structure into, zero-dimensional (0D) e.g., quantum dot /nanoparticles ${ }^{[4-8]}$, one-dimensional (1D) e.g., nanowires and nanorods $(\mathrm{NRs})^{[9-11]}$, and two-dimensional (2D) e.g., nanosheets and nanowalls. ${ }^{[12-15]}$ Compared to 0D and 2D, 1D NSs have the advantage of the possibility of being vertically oriented, which is crucial for many device applications. ${ }^{[16]}$ Among a variety of metal oxides, zinc oxide $(\mathrm{ZnO})$ have a preference of the favorable formation in all NSs forms mentioned above on any substrate being crystalline or amorphous. ${ }^{[9,17-19]}$ Moreover, $\mathrm{ZnO}$ possesses excellent optical and electronic properties. ${ }^{[20-23]}$ All these features make $\mathrm{ZnO}$ as one of the most promising materials for a diversity of applications. In particular, ZnO NRs have recently attracted considerable research interest due to their potential for the development of optoelectronic devices e.g., ultraviolet (UV) photodetectors ${ }^{[24-27]}$, light-emitting diodes (LEDs) ${ }^{[20,22,23]}$ and solar cells. ${ }^{[28]}$ The potential of $\mathrm{ZnO}$ NRs in all these applications, however, would require synthesis of high crystal quality $\mathrm{ZnO}$ NRs with precise control over the morphology, alignment, optical and electronic properties. ${ }^{[22,23,29]}$ Broadly, it is well known that the presence of native (intrinsic) and impurities (extrinsic) defects have a high impact on the electronic and optical properties of $\mathrm{ZnO}$ NRs. ${ }^{[20-23,30-33]}$ In particular, in all 
luminescence processes, these defects in $\mathrm{ZnO}$ are engaged as steps in the excitation and recombination paths, and thus influence the absorbed /emitted light properties. Also, these defects play an important part in the electrical conductivity in semiconductors by the ability to provide delocalized electrons in the conduction band (CB) and holes in the valence band (VB). Consequently, the knowledge of understanding the origin and the control of these defects, as well as the relationship between both, are essential factors for optimizing the optical and electronic properties of $\mathrm{ZnO}$ NRs. ${ }^{[20-23,30-33]}$ However, identifying the nature of such defects is a complex issue, particularly in NSs, where the information on anisotropy is usually lost due to the lack of coherent orientation. ${ }^{[34]}$ To investigate the origin of these defects in more details and to fulfill all the advantages of $\mathrm{ZnO}$ NRs in device applications several synthesis techniques have been utilized. Among them, the low-temperature solution-based methods, which are regarded as promising methods due to many advantages. It is low-cost and offers the possibility of large-scale production and tuning of the properties of the final product through the synthesis parameters. ${ }^{[9,18,35]}$ However, synthesizing reproducible $\mathrm{ZnO}$ NRs with optimized morphology, orientation, electronic and optical properties by the low-temperature solution-based methods remains a challenge.

Thus, the aim of this thesis is towards the optimization of the lowtemperature solution-based synthesis of $\mathrm{ZnO} \mathrm{NRs}$ for device applications. In this connection, we first started with investigating the effect of the precursor solution stirring durations on the deep level defects concentration and their spatial distribution along the $\mathrm{ZnO}$ NRs. Then, by choosing the optimal stirring time, we studied the influence of $\mathrm{ZnO}$ seeding layer precursor's types and its molar ratios on the density of interface defects. The findings of this investigation were used to demonstrate $\mathrm{ZnO}$ NRs- 
based heterojunction LEDs. The ability to tune the point defects along the NRs enabled us further to incorporate cobalt ( $\mathrm{Co}$ ) ions into the $\mathrm{ZnO} \mathrm{NRs}$ crystal lattice, where these ions could occupy vacancies or interstitial defects through substitutional or interstitial doping. Following this, high crystal quality vertically well-oriented $\mathrm{ZnO}$ NRs have been demonstrated by incorporating a small amount of $\mathrm{Co}^{2+}$ into the $\mathrm{ZnO}$ crystal lattice. Finally, the influence of $\mathrm{Co}^{2+}$ ions incorporation on the reduction of coredefects (CDs) in $\mathrm{ZnO}$ NRs was systematically examined using electron paramagnetic resonance (EPR). 


\section{Material characteristics}

\subsection{ZnO characteristics}

Having a direct wide bandgap of about $3.37 \mathrm{eV}$ (at room temperature) with relatively high exciton binding energy $(60 \mathrm{meV})$ and possessing unique luminescence properties enable $\mathrm{ZnO}$ to be an attractive material for many applications. ${ }^{[20,21]}$ Further, $\mathrm{ZnO}$ is considered as one of the most promising semiconductor materials for the development of optoelectronic devices. ${ }^{[20-23]}$ Beside the fact that it is a biocompatible material, $\mathrm{ZnO}$ has a high isoelectric point (IEP 9.5), and exhibits large surface area/volume ratio in its nanostructured forms, and hence is also regarded as excellent material for sensing applications. ${ }^{[36]}$ Moreover, due to the lack of non-central symmetry, $\mathrm{ZnO}$ possesses relatively strong piezoelectric effect, which makes it an attractive material for some energyharvesting applications. ${ }^{[37,38]}$ The fundamental structural, electronic and optical properties of $\mathrm{ZnO}$ is briefly introduced in this chapter.

\subsubsection{Crystal structure}

$\mathrm{ZnO}$ is a member of the II-IV binary compound semiconductor family, and its crystal is either cubic zinc blend or wurtzite-type structure. In the wurtzite structure of $\mathrm{ZnO}$, the $\mathrm{Zn}$ and $\mathrm{O}$ atoms are organized into a hexagonal form where one $\mathrm{Zn}^{2+}$ ion is enclosed tetrahedrally by four $\mathrm{O}^{2-}$ ions and vice versa as shown in the unit cell in Figure 2.1. ${ }^{[21]}$ The unit cell of the hexagonal wurtzite structure of $\mathrm{ZnO}$ has lattice parameters of about $\mathrm{a}=0.32498 \mathrm{~nm}$ and $\mathrm{c}=5.2066 \mathrm{~nm}$ with the ratio of $\mathrm{c} / \mathrm{a}=1.60 .^{[21]}$ Moreover, the hexagonal wurtzite structure of $\mathrm{ZnO}$ is characterized by six nonpolar $\{10 \overline{1} 0\}$ surfaces covered by polar $\mathrm{Zn}(0001)$ and oxygen $(000 \overline{1})$ basal planes. These polar surfaces are electrostatically unstable and hence is leading to the growth habit along the c-axis producing $1 \mathrm{D} \mathrm{ZnO} \mathrm{NRs.}{ }^{[39]}$ 


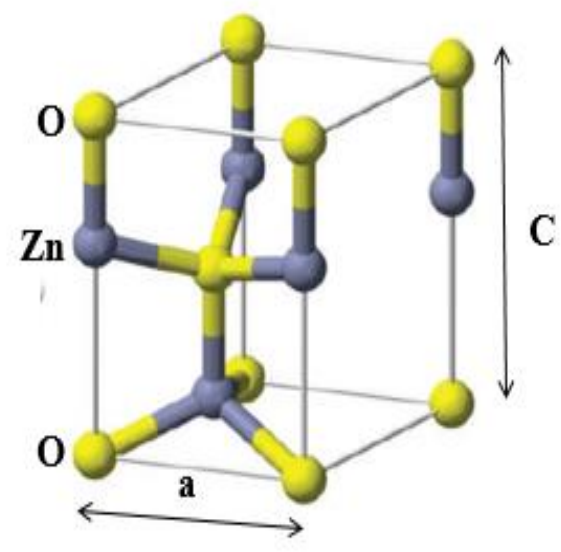

Figure 2.1: The unit cell of the $\mathrm{ZnO}$ hexagonal wurtzite-type structure. ${ }^{[21]}$

\subsubsection{Electronic band structure}

The electronic band gap structure is the most significant property of $\mathrm{ZnO}$, and it is critical when considering it for optoelectronics applications. It is known that at ambient conditions, $\mathrm{ZnO}$ is a direct band gap material where the energy difference $(3.37 \mathrm{eV})$ between the lowermost conduction band (CB) and uppermost valence band (VB) occurs at the same point in the Brillouin zone which is known as the $\Gamma$-point as shown in Figure 2.2. ${ }^{[21]}$ Due to the high ionic feature of $\mathrm{ZnO}$, the bottom of the $\mathrm{CB}$ is principally formed by the $4 \mathrm{~s}$ level of $\mathrm{Zn}^{2+}$ or antibonding $\mathrm{sp}^{3}$ hybrid states, while the $\mathrm{VB}$ is made by the $2 \mathrm{p}$ level of $\mathrm{O}^{2-}$ or the bonding $\mathrm{sp}^{3}$ orbitals. ${ }^{[21]} \mathrm{As}$ predicted by the group theory the bottom of the $\mathrm{CB}$ has a $\Gamma_{1}$ symmetry without the inclusion of spin and symmetry $\Gamma_{1} \otimes \Gamma_{7}=\Gamma_{7}$ with spin as shown in Figure 2.2. The VB is splitted without spin into two states, $\Gamma_{5}$ and $\Gamma_{1}$ under the effect of the hexagonal crystal field of $\mathrm{ZnO}$. Moreover, the inclusion of the spin added further splitting of the VB and provided three twofold-degenerate sub-VBs with the symmetries of $\left(\Gamma_{1} \otimes \Gamma_{5}\right) \otimes \Gamma_{7}=\Gamma_{7}$

$\otimes \Gamma_{9} \otimes \Gamma_{7}$ as shown in Figure 2.2. These three sub-VBs are often labeled 
from the highest to the lowest energies as $\mathrm{A}, \mathrm{B}$, and $\mathrm{C}$, respectively, as illustrated in figure 2.2. ${ }^{[21]}$

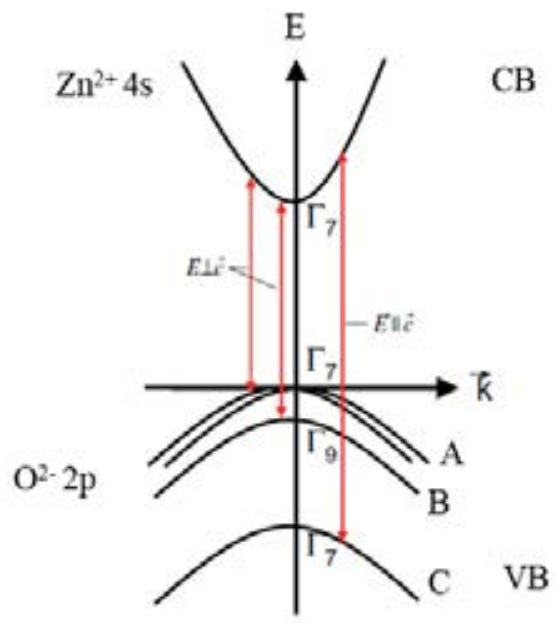

Figure 2.2: Schematic illustration of the $C B$ and $V B$ structure of $\mathrm{ZnO}$ in the vicinity of the fundamental bandgap. ${ }^{[21]}$

\subsubsection{Optical characteristics}

Commonly, it is well known that the optical properties of $\mathrm{ZnO}$ are profoundly affected by the presence of the intrinsic and extrinsic defects. ${ }^{[20-}$ ${ }^{23,30-35]}$ In particular, in all luminescence processes, these defects in $\mathrm{ZnO}$ are involved as steps in the excitation and recombination paths (e.g., radiative and non-radiative recombinations), and thus influencing the absorbed/emitted light properties. The room-temperature luminescence of $\mathrm{ZnO}$ NSs is usually distinguished by a sharp UV emission peak centered at $\sim 380 \mathrm{~nm}$ and attributed to a near-band-edge emission (NBE), and a broad emission peak covers the entire visible region extending between $400-750 \mathrm{~nm}$ as shown in Figure 2.3. ${ }^{[40-44]}$ However, the source of these deep level emissions (DLEs) in the $\mathrm{ZnO}$ is highly controversial, and 
possibly these DLE peaks are composed of multiple sub-bands e.g., green, yellow and orange-red bands. The green emission band centered at $\sim 500$ $-550 \mathrm{~nm}$, is usually ascribed to oxygen vacancy $\left(\mathrm{V}_{\mathrm{O}}\right)$ or zinc vacancy $\left(\mathrm{V}_{\mathrm{Zn}}\right) .{ }^{[20,33,45]}$ The yellow emission band centered between $550-600 \mathrm{~nm}$ and the red emission band centered at $\sim 620-750 \mathrm{~nm}$ are usually assigned the presence of $\mathrm{OH}$ groups and to the oxygen interstitials $\left(\mathrm{O}_{\mathrm{i}}\right)$ present on the surface of the ZnO NRs. ${ }^{[33,40,43,46]}$

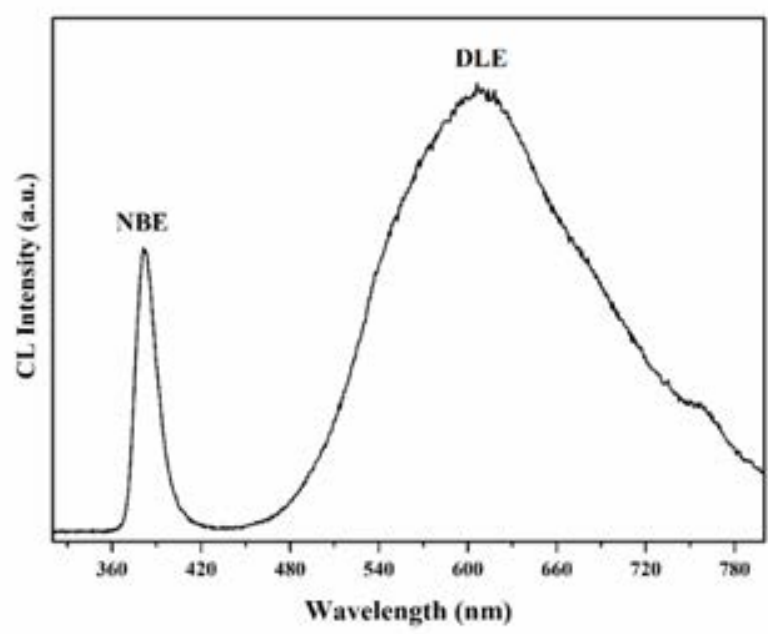

Figure 2.3: Cathodoluminescence (CL) spectrum of ZnO NRs synthesized on a sapphire substrate using the low-temperature aqueous chemical synthesis at $90{ }^{\circ} \mathrm{C}$ from a growth solution of a $0.075 \mathrm{M}$ concentration of hexamethylenetetramine (HMTA) and zinc nitrate hexahydrate in deionized (DI)-water.

\subsection{Co-doped $\mathrm{ZnO}$ characteristics}

Doping is a major method to control the semiconductors properties e.g., optical, electronic and magnetic properties. $\mathrm{ZnO}$ NSs-based diluted magnetic semiconductors (DMSs), where a low concentration of $3 d$ 
transition metals (TMs) ions is diluted into the $\mathrm{ZnO}$ crystal lattice, show great promise for the development of spintronics and magneto-optical devices. ${ }^{[47-50]}$ The potential of this ZnO NRs-based DMSs in spintronics applications would require the presence of the room-temperature ferromagnetism (RT-FM), which is probably related to the successful substitution of these DMSs ions into the $\mathrm{ZnO}$ crystal lattice. ${ }^{[48-50]}$ Beside, the crystal quality, optical and electronic properties of $\mathrm{ZnO}$ NRs-based DMSs together with control over the morphology and alignment is critical in device applications. Among a variety of $3 d \mathrm{TMs}$ ions in ZnO NRs-based DMSs, Co is of particular interest due to its a unique optical and magnetic property. ${ }^{[51,52]}$ Although considerable research progress has been made to synthesize Co-doped $\mathrm{ZnO} \mathrm{NRs}$, most of the research has focused on probing their optical and magnetic properties. ${ }^{[48-51]}$ However, the synthesis of a high crystal quality of Co-doped $\mathrm{ZnO}$ with the successful substitution of $\mathrm{Co}^{2+}$ in the host crystal site is still lacking, which will probably lead to better understanding of the origin of the RT-FM in ZnO. Figure 2.4 (a) shows the electronic configuration of the $3 \mathrm{~d}^{7}$ of $\mathrm{Co}^{2+}$ and (b) illustrates the level diagram of $\mathrm{Co}^{2+}\left(3 \mathrm{~d}^{7}\right)$ ion in $\mathrm{ZnO}$ where first, a free ion (gas): Coulomb interaction leads to ${ }^{4} \mathrm{~F}(\mathrm{~S}=3 / 2, \mathrm{~L}=3)$ ground state, by Hund's rule. Then in a tetrahedral crystal field (case of the nearest neighbors $\mathrm{O}^{2-}$ ions around $\left.\mathrm{Co}^{2+}\right)$, the ground state becomes ${ }^{4} \mathrm{~S}(\mathrm{~S}=3 / 2, \mathrm{~L}=0)$ which is called orbital momentum quenching (by the crystal field). Then by including the spin orbit and the axial part of the crystal field, the ground state is split by $2 \mathrm{D}$, where $\mathrm{D}$ is the axial spin anisotropy parameter, which is positive (easy plane) and it is about $2.5 \mathrm{~cm}^{-1}$. The ground state is then doubly degenerate $(+/-1 / 2$ projection of the $S=3 / 2)$, and the state just above also (+/- $3 / 2$ projection of $S=3 / 2$ ). Finally, taking into account the nuclear spin and the hyperfine interaction each projection is separated into eight levels. 


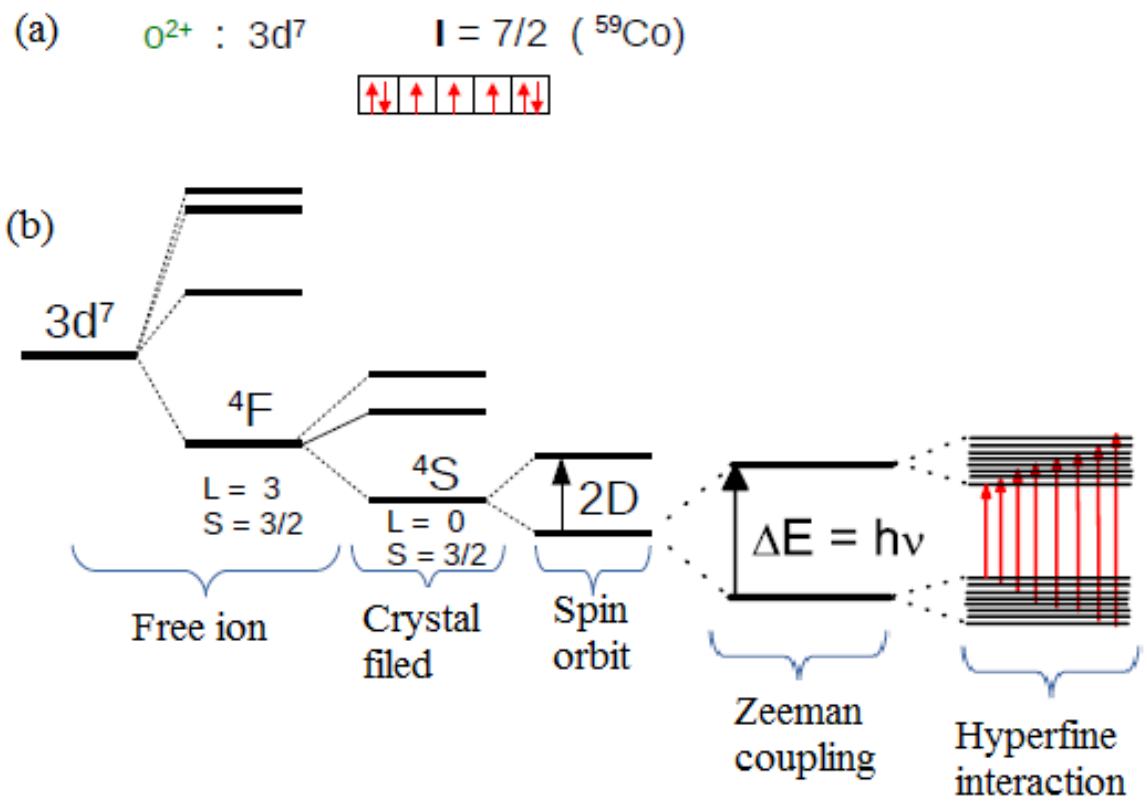

Figure 2.4: (a) Electronic configuration of the $3 d^{7}$ of $\mathrm{Co}^{2+}$. (b) Level diagram of $\mathrm{Co}^{2+}\left(3 d^{7}\right)$ ion in $\mathrm{ZnO}$, under successive applications of free ion, crystal field, spin orbital, Zeeman coupling, and hyperfine interaction. Red arrows depict allowed EPR transitions between non-degenerate hyperfine levels. 


\section{Synthesis methods}

\subsection{Hydrothermal synthesis methods}

Among all the synthesis techniques utilized for $\mathrm{ZnO} \mathrm{NRs}$, the hydrothermal methods are promising due to several advantages. It is lowcost and can be scaled-up for industrial production on any substrates, it is a low-temperature process, and the properties of the final product can be tuned by adjusting the synthesis parameters. ${ }^{[9,18,35]}$ The hydrothermal term refers to the synthesis methods that are carried out in an aqueous solution. The chemistry of synthesis of $\mathrm{ZnO}$ NRs in the aqueous solution methods is well studied, and the most common recipes used as a reaction material is hydrolysis of zinc nitrate in water in the presence of hexamethylenetetramine (HMTA). ${ }^{[19,41,53-55]}$ First, zinc nitrate is dissolved into water giving rise to aquo ions containing different $\mathrm{Zn}$ (II) hydroxyl species. Then at a particular concentration of $\mathrm{Zn}$ (II), the stability of these complexes hydroxyl species depend on the temperature and $\mathrm{pH}$ of the solution. After that, the solid $\mathrm{ZnO}$ nuclei will then be formed by the dehydration of these hydroxyl species, and subsequently, the $\mathrm{ZnO}$ crystal continues to grow by the condensation of the surface hydroxyl groups with zinc-hydroxyl complexes. ${ }^{[53-55]}$ The hydrolysis and condensation reactions will then result in $1 \mathrm{D} \mathrm{ZnO}$ crystals under a wide diversity of synthesis conditions e.g., $\mathrm{pH}$, concentration, temperature, time. On the other hand, the HMTA plays various important roles during the synthesis process. ${ }^{[19,35,53-55]}$ It is highly accepted that the HMTA supplies the $\mathrm{OH}^{-}$ during the $\mathrm{ZnO}$ NRs synthesis process by pushing the precipitation reaction through thermal degradation. Beside the fact that the HMTA function as a $\mathrm{pH}$ buffer by slow hydrolysis and hence producing ammonia $\left(\mathrm{NH}_{3}\right)$ and formaldehyde as shown in equations 3.1 and 3.2, respectively. 
[19,53-55] Also, it is proposed that during the synthesis process of $\mathrm{ZnO}$ crystal, the HMTA attaches to the nonpolar surface of $\mathrm{ZnO}$ NRs and hence prohibit the access of the $\mathrm{Zn}^{2+}$ to them and therefore leaving only the polar surface for epitaxial growth. The chemical reactions for obtaining ZnO NSs in an aqueous solution when zinc nitrate and HMTA are used as a reaction material can be summarized in the following equations: ${ }^{[53-55]}$

Decommission reaction:

$$
\mathrm{C}_{6} \mathrm{H}_{12} \mathrm{~N}_{4}+6 \mathrm{H}_{2} \mathrm{O} \leftrightarrow 4 \mathrm{NH}_{3}+6 \mathrm{HCHO}
$$

Hydroxyl supply reaction:

$\mathrm{NH}_{3}+\mathrm{H}_{2} \mathrm{O} \leftrightarrow \mathrm{NH}_{4}^{+}+\mathrm{OH}^{-}$

Super-saturation reaction:

$\mathrm{Zn}^{2+}+2 \mathrm{HO}^{-} \rightarrow \mathrm{Zn}(\mathrm{OH})_{2}$

ZnO NRs synthesis reaction:

$$
\mathrm{Zn}(\mathrm{OH})_{2} \rightarrow \mathrm{ZnO}_{(\mathrm{S})}+\mathrm{H}_{2} \mathrm{O}
$$

Controlling all these reactions play a significant effect on the final properties of the synthesized $\mathrm{ZnO}$ NSs. ${ }^{[19,55]}$

In this thesis, pure and Co-doped ZnO NRs were synthesized by the low-temperature aqueous synthesis (LT-ACS) at $80-90{ }^{\circ} \mathrm{C}$ on silver (Ag) coated glass, p-type GaN, and sapphire substrates, respectively. In the experiments, we mainly investigated the synthesis conditions e.g., stirring durations of the synthesize solutions, $\mathrm{ZnO}$ seeding layer precursors types, and its molar ratios to obtain vertically-well aligned $\mathrm{ZnO}$ NRs with tuned defects emission along the NRs and at the interfaces between the NRs and the substrates. ${ }^{[56-58]}$ Moreover, high crystal quality well-oriented Co-doped 
$\mathrm{ZnO}$ NRs were also synthesized by manipulating the way of mixing the synthesizer solution. ${ }^{[34,52,59]}$ All these parameters were precisely selected considering that the final properties of the $\mathrm{ZnO}$ NRs are susceptible to the synthesis conditions. A typical LT-ACS of ZnO NSs consists of two main steps: first, a clean substrate is subjected to spin coating with a $\mathrm{ZnO}$ seed layer and then $\mathrm{ZnO}$ NSs are subsequently, synthesized from a zinc ion containing solution. It is to be noted that before these two steps, all substrates used in this work were first cleaned with acetone, isopropanol, and deionized water (DI-water) for 5 minutes each to eliminate any residuals, and were then dried up using blowing nitrogen. We found that these steps play a significant role in the final structural and optical quality of the synthesized $\mathrm{ZnO}$ NRs.

\subsection{ZnO seed layer preparation}

In the LT-ACS it is widely accepted that the presence of the seed layer is required to promote nucleation sites for $\mathrm{ZnO}$ NRs synthesis by lowering the thermodynamic barrier between heterogeneous materials. ${ }^{[35]}$ Also, it is known that the properties of the seed layer have a significant impact on the quality of $\mathrm{ZnO}$ NRs synthesis and consequently on their final properties. ${ }^{[60-65]}$ In general, the seed layer can be prepared in the form of thin film (TF) or nanoparticles (NPs) and then can be deposited on a certain substrate by several deposition techniques e.g., sputtering, spin coating and dip coating. ${ }^{[18,35,60]}$ In particular, the NPs seed layer is often prepared by the dissolution of zinc acetate dihydrate $\left(\mathrm{Zn}\left(\mathrm{CH}_{3} \mathrm{COO}\right)_{2} \cdot \mathrm{H}_{2} \mathrm{O}\right)$ in organic solvents e.g., methanol, and the subsequent addition of a basic aqueous solution e.g., potassium hydroxide $(\mathrm{KOH}) .{ }^{[35,53]} \mathrm{In}$ this thesis, two types of seed layers were prepared, and their effect on the performance of the synthesized ZnO-based heterojunction LEDs was systematically 
studied. ${ }^{[57,58]}$ The first seed layer is made by dissolution $0.01 \mathrm{M}$ concentration of zinc (II) acetate dihydrate in methanol and then heated at $60{ }^{\circ} \mathrm{C}$ on a hotplate under continuous stirring. Subsequently, a solution of $\mathrm{KOH}$ in methanol with different concentrations of $0.01,0.03$ and $0.05 \mathrm{M}$, respectively, was added dropwise to zinc (II) acetate solution. Then the whole solution was kept on a hotplate for 2 hours (h) ${ }^{[57]}$ Figure 3.1 shows the atomic force microscopy (AFM) image of the prepared seed layer with molar ratios of 1:1, 1:3 and 1:5 M, respectively. The second seed layer was made by following the same procedure used for the previous one where a mixture of $\mathrm{KOH}$ and HMTA with $0.05 \mathrm{M}$ concentration were dissolved in methanol and were then added dropwise to a prepared $0.01 \mathrm{M}$

concentration of zinc (II) acetate dihydrate in methanol. ${ }^{[58]}$ For the synthesis of the pure and Co-doped $\mathrm{ZnO} \mathrm{NRs}$, the prepared seed layer solutions were then spun coated 3-4 times on the substrates with a spin speed of 3000 round per minute (rpm) for 3 minutes. This step was then followed by annealing in a normal laboratory oven for 10 minutes at 120 ${ }^{\circ} \mathrm{C}$ and subsequently submerged in the $\mathrm{ZnO}$ synthesis solution. Finally, the synthesized ZnO NRs on the seeded substrates were rinsed with DI-water to remove any residuals and were finally dried using blowing nitrogen for further characterization.

\subsection{Synthesis of $\mathrm{ZnO}$ NRs}

After optimization of the many synthesis parameters, to achieve highly dense and vertically aligned $\mathrm{ZnO}$ NRs with tuned optical properties. The following conditions were utilized to synthesize the ZnO NRs in the entire thesis. The synthesis solutions used were $0.05 \mathrm{M}$ concentration for the zinc nitrate and $0.075 \mathrm{M}$ concentration for the HMTA with a molar ratio of 1:2. ${ }^{[56-58]}$ Then we investigated the effect of stirring durations of the 
synthesis solutions on the optical properties of the ZnO NRs. ${ }^{[56]}$ Four synthesis precursor's (solution) consisting of zinc nitrate and HMTA were dissolved separately in $100 \mathrm{ml}$ DI-water each and were then carefully mixed and stirred at room-temperature for 1, 3, 5 and $15 \mathrm{~h}$, respectively, with a stirring speed of $600 \mathrm{rpm}$. The $\mathrm{ZnO}$ NRs were then synthesized by immersing a seeded glass substrate coated with $\mathrm{Ag}$ inside the synthesis solution while keeping them in a preheated oven at $80{ }^{\circ} \mathrm{C}$ for $6 \mathrm{~h}$. Afterward, the samples were cleaned with DI-water to remove any residuals and were finally dried using blowing nitrogen.
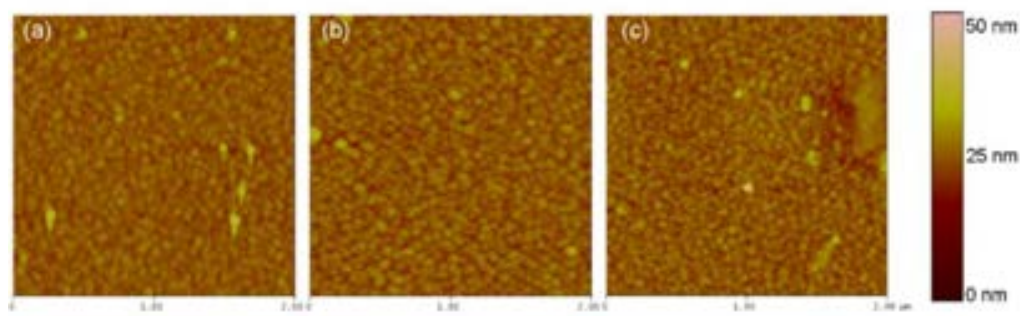

Figure 3.1: Surface topography of the prepared seed layer using zinc (II) acetate dihydrate and $\mathrm{KOH}$ with molar ratios of (a ) 1:1, (b) 1:3, and (c) 1:5 M. Scanned area: $2 \times 2 \mu \mathrm{m}$.

Figure 3.2 shows the top-view FE-SEM image of the synthesized $\mathrm{ZnO}$ NRs. The effect of stirring durations on the concentration and spatial distribution of the deep level defects in the synthesized $\mathrm{ZnO}$ NRs were systematically studied. ${ }^{[56]}$

\subsection{Synthesis of Co-doped ZnO NRS}

For the synthesis of high crystal quality well-oriented pure and Co-doped $\mathrm{ZnO} \mathrm{NRs}$, two different processes for preparing the synthesis solution were used. ${ }^{[34,52,59]}$ The first process for preparing the synthesis 
solution is carried out by making a $0.075 \mathrm{M}$ concentration of HMT separately in $100 \mathrm{ml}$ of DI-water and subsequently stirred at roomtemperature for $1 \mathrm{~h}$. Then, diluted solutions of Cobalt (II) acetate tetrahydrate in $60 \mathrm{ml}$ of DI-water with different atomic concentrations were mixed with the HMTA solution and stirred for $15 \mathrm{~h}$. After that, a $0.075 \mathrm{M}$ concentration of zinc nitrate hexahydrate prepared in $40 \mathrm{ml}$ of DIwater was added dropwise to the solutions mixed above and stirred for additional $3 \mathrm{~h}$. Finally, the seeded sapphire substrates were then submerged vertically inside the synthesis solutions and kept in the oven at $90{ }^{\circ} \mathrm{C}$ for 6 h. After the NRs growth, the samples were rinsed with DI-water to remove any residuals and finally dried using blowing nitrogen. ${ }^{[59]}$ The second process was done by mixing a $0.075 \mathrm{M}$ concentration of zinc nitrate, and HMTA prepared separately in $70 \mathrm{ml}$ DI-water each and were then mixed and stirred for $15 \mathrm{~h}$. Then, diluted solutions of Cobalt (II) nitrate hexahydrate in $40 \mathrm{ml}$ DI-water with different atomic concentrations was added dropwise to the solution above and stirred for further $3 h^{[34,52,59]}$

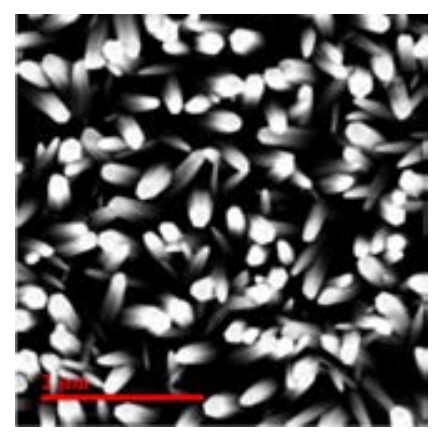

Figure 3.2: Top view FE-SEM images of the synthesized pure NRs grown using $0.05 \mathrm{M}$ concentration of zinc nitrate and $0.075 \mathrm{M}$ concentration of HMTA at $80{ }^{\circ} \mathrm{C}$ for $6 \mathrm{~h}$. 
The influence of these two processes in the incorporation of $\mathrm{Co}^{2+}$ inside the ZnO NRs crystal lattice and the subsequent effect of them on the crystal quality, orientation, and defects properties were systematically investigated. ${ }^{[34,52,59]}$ Figure 3.3 shows the synthesized Co-doped ZnO NRs by using the first and second approach for preparing the synthesis solution.

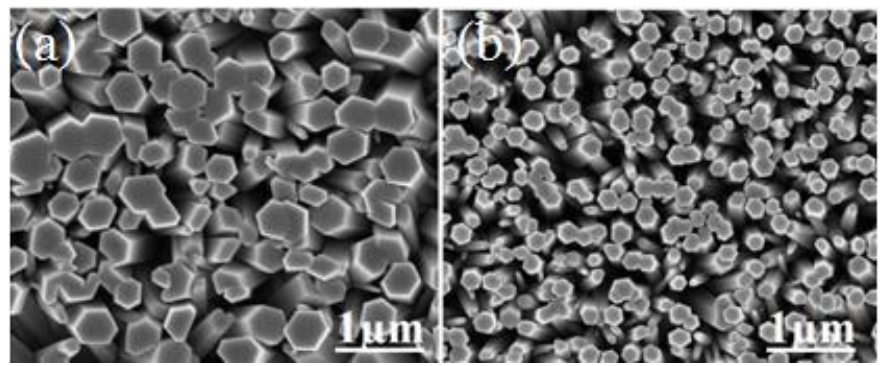

Figure 3.3: Top-view FE-SEM images of the synthesized Co-doped $\mathrm{ZnO}$ NRs grown using the first (a), and (b) second method for preparing the synthesis solution, respectively.

\subsection{N-ZnO NRs/p-GaN-based heterojunction LEDs}

At this stage, a successful synthesis of $\mathrm{ZnO}$ NRs with tunable optical properties using a seed layer prepared with different precursors solution and molar ratios was achieved. Further, the exploration of their potential application in LEDs was investigated. The $n-\mathrm{ZnO} \mathrm{NRs} / \mathrm{p}-\mathrm{GaN}-$ based heterojunction LEDs in this thesis was fabricated using a commercially available p-type GaN (which were magnesium-doped GaN (0001)-oriented layer grown on sapphire purchased from universitywafer from the U.S.A.). The fabrication process of the $\mathrm{n}-\mathrm{ZnO} \mathrm{NRs} / \mathrm{p}-\mathrm{GaN}-$ based heterojunction LEDs is described as follow: the synthesized n-ZnO NRs/p$\mathrm{GaN}$ heterostructures were first, spun coated with an insulating layer of Shipley 1805 photoresist to electrically isolate the ZnO NRs from the p$\mathrm{GaN}$ substrates and then backing in $110^{\circ} \mathrm{C}$ for $1-3$ minutes. Reactive ion 
etching (RIE) with oxygen plasma was then used to remove the photoresist from the $\mathrm{ZnO}$ NRs surface and expose the NRs tip for the metal contacts. Finally, $\mathrm{Ni} / \mathrm{Au}(15 \mathrm{~nm} / 35 \mathrm{~nm})$ were thermally evaporated onto the $\mathrm{p}-\mathrm{GaN}$ substrates and $\mathrm{Ag}(40 \mathrm{~nm})$ on the exposed $\mathrm{ZnO}$ NRs tip to serve as p-type and n-type contact electrodes, respectively. ${ }^{[57,58]}$ The p-type and n-type contacts were evaporated in a circular area of diameter $\sim 2 \mathrm{~mm}$. The fabrication process is illustrated in Figure 3.4.
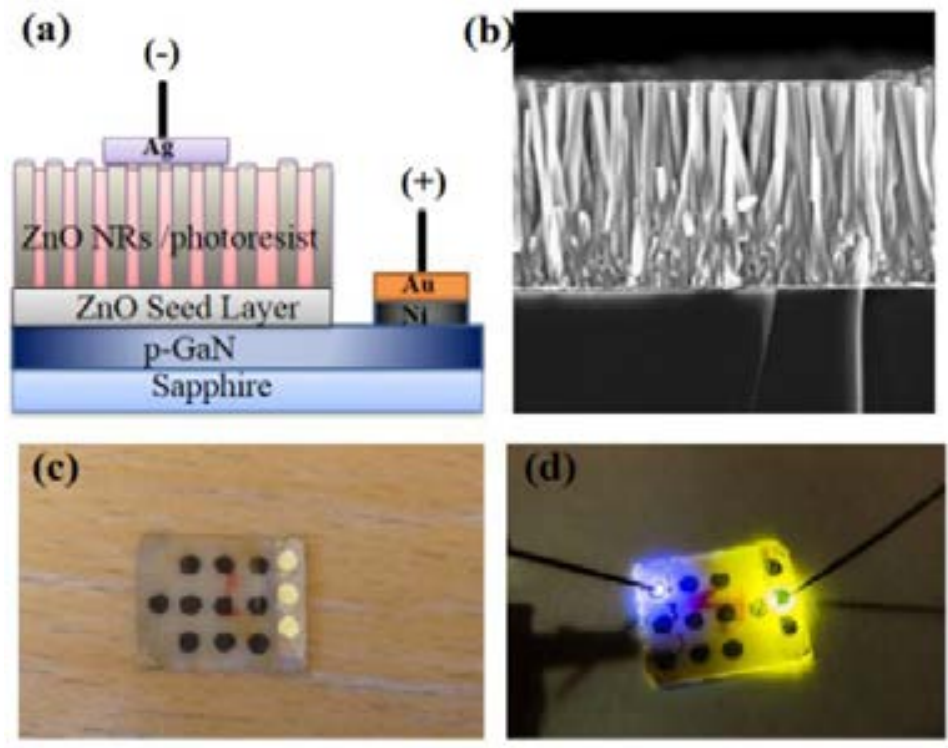

Figure 3.4: (a) Schematic diagram illustrating the fabricated heterojunction LEDs, (b) Cross-sectional SEM image of the synthesized n$\mathrm{ZnO}$ NRs on the p-GaN substrate, (c) Optical photograph of the fabricated heterojunction LEDs on a p-GaN substrate, and (d) Optical photograph of the fabricated heterojunction LEDs under forwarding bias. 


\section{Characterization tools}

Several characterization tools have been employed to get insights into morphology and the structural, optical, electrical and electronic properties of the synthesized pure and Co-doped $\mathrm{ZnO}$ NRs. Fieldemission-scanning electron microscopy (FE-SEM) was utilized to characterize the morphological properties of the synthesized pure and Codoped $\mathrm{ZnO}$ NRs. The crystalline quality and electronic structure were investigated using the $\mathrm{x}$-ray diffraction (XRD) and electron paramagnetic resonance (EPR), respectively. Also, EPR was applied to confirm the successful substitution of $\mathrm{Co}$ inside the $\mathrm{ZnO}$ crystal lattice. Then, microphotoluminescence $(\mu-\mathrm{PL})$, cathodoluminescence $(\mathrm{CL})$, and $\mathrm{x}$-ray photoelectron spectroscopy (XPS) were used to gain detailed information on defects emission and their spatial distribution in the synthesized NRs. Electroluminescence (EL) and current-voltage (I-V) measurements were performed to study the light emission and electrical properties of the processed $\mathrm{n}-\mathrm{ZnO} \mathrm{NRs} / \mathrm{p}-\mathrm{GaN}$-based heterojunction LEDs, respectively. It is to be noted that all these measurements were carried out at roomtemperature (RM) except for the EPR measurements, which were done at 5-6 K. A brief introduction to the working principle to all these analytical tools is given below.

\subsection{Scanning electron microscopy (SEM)}

Scanning electron microscopy (SEM) is a helpful instrument to characterize the morphology of a material that is either in the NSs or TF forms. ${ }^{[66]}$ In the SEM, high-resolution secondary or backscattered electrons image of the nanostructured sample is collected as a result of the interaction of electron beam bombardment (which is in the range of several $\mathrm{keV}$ ) with the sample surface. ${ }^{[66]}$ In this thesis, FE-SEM Gemini LEO 1550 was used 
to examine the morphology of the synthesized pure and Co-doped $\mathrm{ZnO}$ NRs.

\subsection{X-ray diffraction (XRD)}

X-ray diffraction (XRD) is a non-destructive characterization technique widely used to study the crystal structure of single or polycrystalline materials. A monochromatic beam of $\mathrm{x}$-rays with a specific wavelength, $\lambda$, is generated by accelerating electrons towards a metal target e.g., copper $(\mathrm{Cu})$. The emitted x-rays strike the material under study at an incidence angle, $\theta$, and the diffracted radiation is collected in a detector at an angle of $2 \theta$ from the incident beam (see Figure 4.1). At certain diffraction angles the x-rays come out in phase e.g., they are coherently scattered, and this will yield a constructive interference, and high intensity is observed at the detector. At these angles, the diffraction condition and the so-called Bragg's law, $n \lambda=2 d \sin \theta$, where $n$ is an integer recognized as the diffraction order, and $d$ is the distance between the diffraction planes, is fulfilled. By analyzing the measured diffraction pattern valuable information on the crystal structure, crystal orientation, phase content, and chemical composition of the material under study can be obtained. ${ }^{[67]}$ In this thesis, the XRD measurements were performed using a Philips PW1729 diffractometer equipped with $\mathrm{Cu} \mathrm{K}_{\alpha}$ radiation $(\lambda=1.54 \AA)$ operated at $40 \mathrm{kV}$ and $40 \mathrm{~mA}$ to investigate the crystal quality of the synthesized pure and Co-doped $\mathrm{ZnO}$ NRs. 


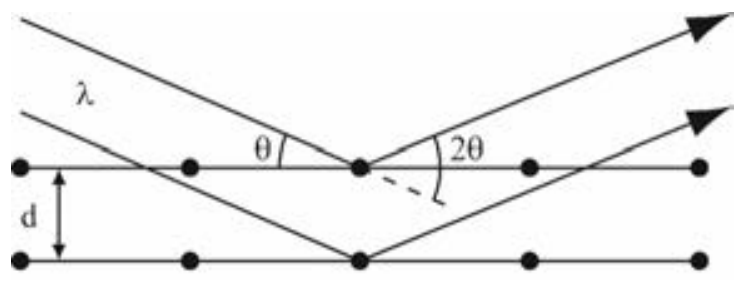

Figure 4.1: Schematic illustration of the principle in the $x$-ray diffraction.

\subsection{X-ray photoelectron spectroscopy (XPS)}

X-ray photoelectron spectroscopy (XPS) is a robust surface analysis technique which is widely utilized for the identification of chemical composition and electronic structure of the material. ${ }^{[68,69]}$ Based on the photoelectric effect, the sample is irradiated by x-ray beam with a known photon energy under ultra-high vacuum (UHV) conditions, the emitted photoelectrons with different kinetic energies from a material surface will be measured (see Figure 4.2). Correspondingly, their binding energy then can be calculated by Einstein equation. Each element present in the material surface has a unique electronic structure and will produce a unique set of peaks (a fingerprint) with recognized emission lines, and by comparing these emission lines to the reference XPS database, different species in the material can be identified. ${ }^{[68]}$ In this thesis, XPS measurements were recorded utilizing Scienta ESCA-200 spectrometer with a monochromatic $\mathrm{Al} \mathrm{K}_{\alpha} \mathrm{x}$-ray source $(1486.6 \mathrm{eV})$ to determine the chemical composition of the material. This technique has also been used to determine and estimate the defects concentrations present in the synthesized pure and Co-doped $\mathrm{ZnO} \mathrm{NRs}$, respectively. The results of the XPS measurements is discussed in detail in the papers included in this thesis. 


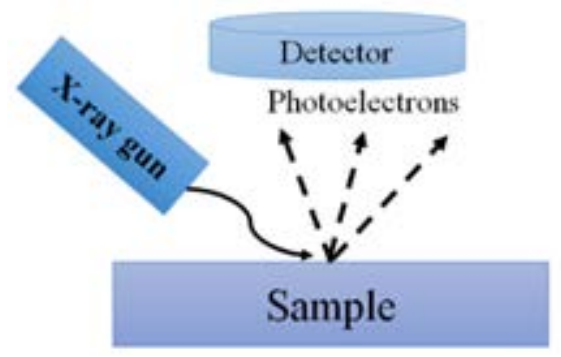

Figure 4.2: Schematic illustration of the XPS experiment.

\subsection{Photoluminescence (PL) spectroscopy}

Photoluminescence (PL) is a powerful optical spectroscopy technique, used to characterize the emission of photons from a fluorescent material upon excitation by light photons (usually laser) of a certain wavelength. ${ }^{[70]}$ The light energy should be above the band gap of the material to excite the carriers (electrons/holes) from the VB to the CB. PL then occurs due to the direct radiative recombination of electrons and holes with the emission of photons, possessing the energy equal to the band gap of the fluorescent material under study. In this thesis, PL is used to characterize the light emission of the synthesized $\mathrm{ZnO}$ NRs in the range $350-800 \mathrm{~nm}$ using a $266 \mathrm{~nm}$ continuous wave laser source as excitation.

\subsection{Cathodoluminescence (CL) spectroscopy}

Cathodoluminescence (CL) is a powerful advanced optical spectroscopy technique used to characterize the emission of photons from a fluorescent material that is exposed to electron beam bombardment. ${ }^{[71-75]}$ Only a small part of the total energy (see Figure 4.3) is carried by the electron beam (which is in the range of several $\mathrm{keV}$ ) is needed to promote excitation of an electron from the VB to the $\mathrm{CB}$, which corresponds to the band gap energy in the range of several $\mathrm{eV}$. CL occurs then due to 
recombination of electrons and holes with the radiation of photons. ${ }^{[76]}$ The CL and PL spectroscopy are similar, however, the level of excitation is different since in PL one photon excites typically one electron-hole pair, while in the CL, one electron of high energy can excite thousands of electron-hole pairs. Also, CL has such advantages of usually providing a better depth-resolved information and a better spatial resolution $(<1$ $\mu \mathrm{m}) .{ }^{[71-75]}$ In this thesis, CL is used to investigate the point defects concentration from aggregates and along the synthesized $\mathrm{ZnO}$ NRs using an acceleration voltage of $5 \mathrm{kV}$ using a Gatan Mono CL4 system combined with Gemini LEO1550 FE-SEM.

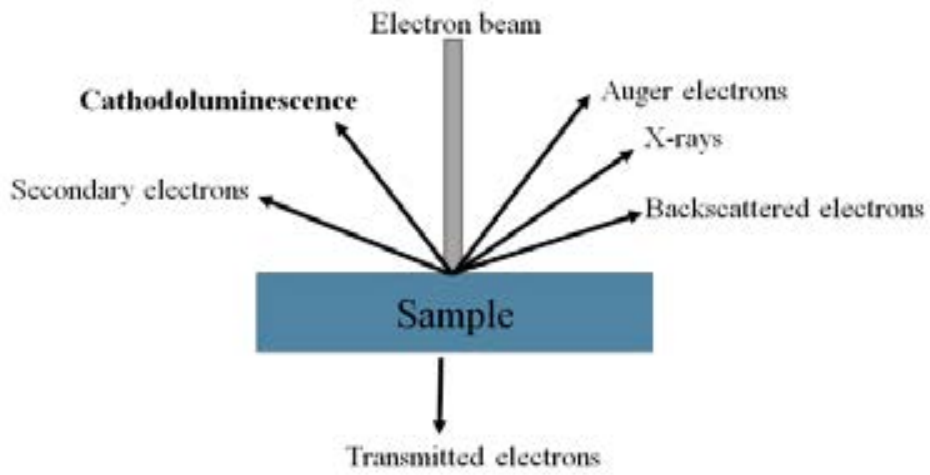

Figure 4.3: Schematic illustration of the signals from the sample under electron beam bombardment. ${ }^{[75]}$

\subsection{Current -Voltage (I-V) measurements}

The electrical properties of the as processed heterojunction LEDbased on the synthesized $\mathrm{n}-\mathrm{ZnO} \mathrm{NRs} / \mathrm{p}-\mathrm{GaN}$ in this thesis were measured using multiple sources-measurement units (SMUs) semiconductor parameter analyzer (Keithley 2400-SCS). In which the p-and n-metal contacts (electrodes) of the fabricated heterojunction LEDs were 
connected to SMUs through a coaxial cable. Then the LED current response is measured as the voltage sweeps in a constant period.

\subsection{Electroluminescence (EL) measurements}

Electroluminescence (EL) measurements is a powerful electrooptical technique widely used to investigate the emitted light from LEDs when electrical current is applied. The electrical current drives the carriers (holes/electrons) across the LED. EL then occurs due to the electrons and holes radiative recombination within the diode leading to the emission of photons. In this thesis, EL measurement is used to characterize the light emission from the fabricated $\mathrm{n}-\mathrm{ZnO} \mathrm{NRs} / \mathrm{p}-\mathrm{GaN}$-based heterojunction LEDs. The EL measurements were performed using a Keithley 2400 source to provide a fixed voltage, while the emission spectra were collected using an SR-303i-B detection system. Figure 4.4 shows a typical EL from the fabricated $\mathrm{ZnO} \mathrm{NRs} / \mathrm{p}-\mathrm{GaN}-$ based heterojunction LEDs.

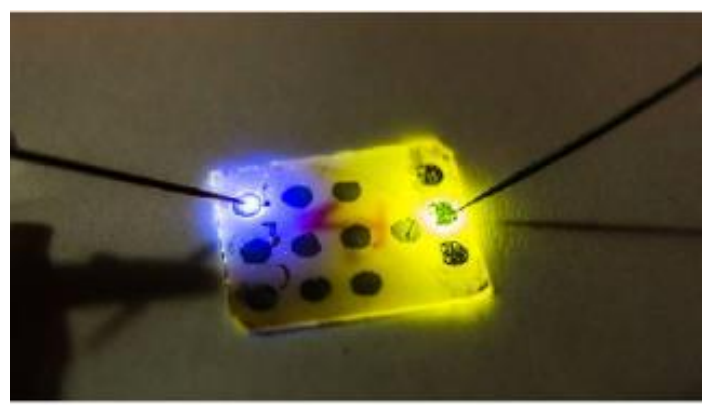

Figure 4.4: Optical photograph illustrating the light emission from the fabricated ZnO NRs/ GaN heterojunction LED under forwarding bias.

\subsection{Electron paramagnetic resonance (EPR) measurements}

Electron paramagnetic resonance (EPR) is a valuable characterization tool that offers a unique way to investigate the ground 
states of localized magnetic impurities in a material and, in turn, to deduce many structural, electronic and magnetic information on these impurities and the material itself. ${ }^{[34,52,77]}$ In a typical X-band EPR experiment, a paramagnetic material is placed within a resonant cavity and in a quasistatic magnetic field $(\boldsymbol{B})$ which split a degenerated 1/2 spin level into its two-spin projection $-1 / 2$ and $+1 / 2$. Then magnetic field $(\boldsymbol{B})$ is slowly varied from 0 to $1.4 \mathrm{~T}$, and at the same time, a continuous stationary microwave of constant energy (hv) is sent into the cavity as illustrated in Figure 4.5. When the Zeeman splitting matches the photon energy ( $h v)$, and if some appropriate selection rules are satisfied, the paramagnetic sample can absorbs this energy. The magnetic field at which this absorption occurs is determined by the EPR line. Then with a deep knowledge of analyzing the energy differences $(\Delta \mathrm{E})$, line width, density, and position, one can gain insight into the identity, structure, and magnetic properties of the material under study. ${ }^{[34,52]}$

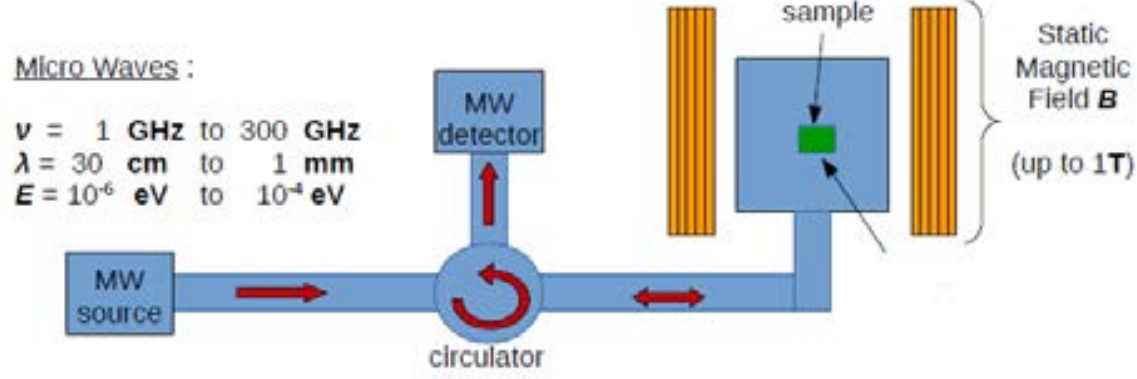

Figure 4.5: Schematic illustration of the EPR experiment.

In this thesis, EPR measurements were performed to investigate the crystal quality, orientation and to give insight into point defects that are present in the synthesized pure and Co-doped $\mathrm{ZnO}$ NRs. The EPR measurements were carried out by a conventional Bruker ELEXSYS continuous wave spectrometer operating at $X$-band $(v=9.38 \mathrm{GHz})$ 
equipped with a standard $\mathrm{TE}_{102}$ mode cavity. The angle between the static magnetic field $(\boldsymbol{B})$ and the $\mathrm{ZnO}$ NRs axis, denoted by $\theta$, was monitored by a manual goniometer. ${ }^{[52]}$ Figure 4.6 shows the illustration of the ZnO NRs samples and definition of the angle, $\theta$, between the $\boldsymbol{B}$ and the c-axis of the NRs.
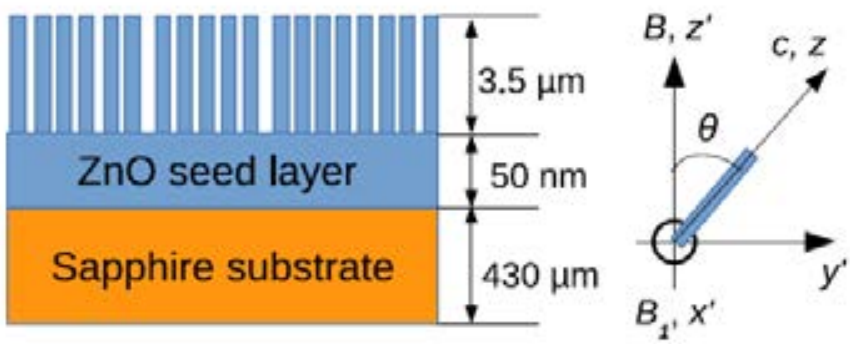

Figure 4.6: Schematic illustrations of the NRs samples and definition of the angle, $\theta$, between the magnetic field $B$ and the c-axis of the NRs. The microwave magnetic field $B_{1}$ is perpendicular to both $B$ and $C$. 


\section{Results and discussion}

The effect of the parameters of the low-temperature aqueous chemical synthesis e.g., stirring time, $\mathrm{ZnO}$ seeding layer precursor's types, and its molar ratios, and the way of mixing the synthesis solution on the properties of the synthesized pure and Co-doped $\mathrm{ZnO}$ NRs have been systematically investigated in this thesis. Below are a description and discussion of these results that were published in the six appended papers. $^{[34,52,56-59]}$

\subsection{The effect of precursor (synthesis) solution stirring durations on the optical properties of $\mathrm{ZnO} \mathrm{NRs}$}

In the low-temperature solution-based synthesis of $\mathrm{ZnO} \mathrm{NRs,} \mathrm{stirring}$ of the precursor (synthesis) solution in the preparation steps are critical to enhance and control the chemical homogeneity of the ionic species. Hence, an optimized stirring would further increase the synthesis rate by lowering the effect of the activation energy during the synthesis process of $\mathrm{ZnO}$ NRs. ${ }^{[78,79]}$ Moreover, stirring the precursor solution could in a way control the concentration of the $\mathrm{OH}^{-}$and consequently the super-saturation reaction under thermodynamic equilibrium conditions during the synthesis process of the $\mathrm{ZnO}$ NRs. Based on the importance of the stirring on the synthesis of $\mathrm{ZnO}$ NRs we have been motivated to investigate the effect of stirring durations of the synthesis solution as a possible way to tune the radiative point defects in the $\mathrm{ZnO} N \mathrm{NRs}^{[56]}$ The main observations in this study is found that stirring durations have a significant influence on the morphology and point defects concentrations and their spatial distribution along the synthesized $\mathrm{ZnO}$ NRs. We observed that the diameters of the synthesized $\mathrm{ZnO}$ NRs were increased from 120, 180 and to $300 \mathrm{~nm}$ as the stirring durations were increased from 1,3 to $5 \mathrm{~h}$, respectively, as shown 
in Figure 5.1 (a) - (c). Moreover, a decrease in the NRs diameter to the 200 $\mathrm{nm}$ was observed upon increasing the stirring durations further to $15 \mathrm{~h}$, as shown in Figure 5.1 (d). The significant difference between the NRs diameters upon stirring durations is most likely believed to be related to the super-saturations state of the $\mathrm{Zn}^{2+}$ and $\mathrm{OH}^{-}$species during the NRs synthesis process. ${ }^{[41,53]}$

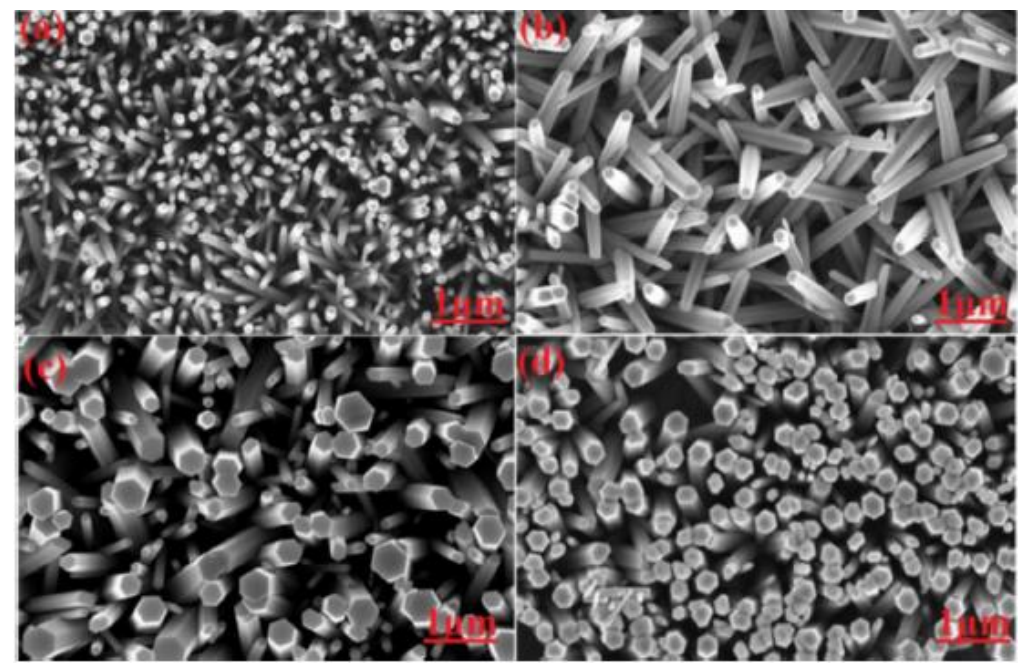

Figure 5.1: Top view FE-SEM images of the synthesized ZnO NRs prepared under stirring durations of (a) $1 h$, (b) $3 h$, (c) $5 h$, (c), and (d) $15 \mathrm{~h}$, respectively.

The synthesis process of $\mathrm{ZnO}$ NRs in the solution-based methods is considered to be controlled by high and low super-saturation reactions. High super-saturation reactions favor nucleation, while low supersaturation reactions favor the synthesis process. ${ }^{[53-55]}$

Figure 5.2 (a)-(d) show a spatially resolved CL spectra collected from individual $\mathrm{ZnO} \mathrm{NR}$ in cross-sectional view at different points along the NR as defined by colored squares in the insert. The CL spectra of all 
samples were characterized by UV emission peak centered at $\sim 382 \mathrm{~nm}$ due to NBE emission and dominated by a broad yellow-orange emission centered at $\sim 610 \mathrm{~nm}$ linked with deep-level (DL) defects associated emission in $\mathrm{ZnO}^{[40-46]}$ Interestingly, the CL intensity of the DL defect emissions along the NRs showed stirring durations dependence as presented in Figure 5.2. As shown in Figure 5.2 (a)-(b), the intensity of the DL defects emission along the NRs was observed to be improved when moving from the bottom of the NR to the top as the stirring durations were varied from 1 to $3 \mathrm{~h}$. Further, as the stirring durations were further increased from 5 to $15 \mathrm{~h}$, the intensity of the DL emission peaks were seen to be reduced while moving toward the tip of the NR as displayed Figure 5.2 (c)-(d). As suggested above the control of the super-saturation of the $\mathrm{OH}^{-}$reactions are significant during the synthesis process. When a relatively high amount of $\mathrm{OH}^{-}$is produced in a short period, the $\mathrm{Zn}^{2+}$ in the synthesis solution will precipitate out rapidly due to the high $\mathrm{pH}$ environment. Consequently, $\mathrm{Zn}^{2+}$ would contribute little to the $\mathrm{ZnO} \mathrm{NRs}$ synthesis and eventually result in a quick consumption of the $\mathrm{Zn}$ precursor and prohibit the further growth of the $\mathrm{ZnO}$ NRs. ${ }^{[19]}$ Therefore, it is highly possible that the stirring of the precursor solution for 1 and $3 \mathrm{~h}$ in the preparation step promote high super-saturation under thermodynamic equilibrium during the synthesis process. Consequently, the synthesis solution becomes weak in $\mathrm{Zn}$, and hence favoring the creation of point defects going downward the tip of the NR as presented in Figure 5.2 (a)(b). In contrary, stirring for 5 and $15 \mathrm{~h}$ favors low super-saturation during the synthesis process of the NRs, and consequently, the synthesis solution becomes rich in $\mathrm{Zn}$, assisting the formation of point defects moving toward the base of the NR as shown in Figure 5.2 (c)-(d). 
The phenomenon of the altered CL intensity with the stirring durations can be explained using results of first-principle density functional theory calculations, the formation energy of the point defects in semiconductors depend on the synthesis conditions. In zinc-rich conditions, $\mathrm{V}_{\mathrm{O}}$ has lower formation energy than zinc interstitial $\left(\mathrm{Zn}_{\mathrm{i}}\right)^{[30,31]}$, which is most probably the case in our low-temperature aqueous solution synthesis because the $\mathrm{O}$ comes from the super-saturation of the $\mathrm{OH}^{-}$ reactions, while the zinc nitrate provides the $\mathrm{Zn}$ in the solution. ${ }^{[35]}$ The point defects in our synthesized $\mathrm{ZnO}$ NRs are most likely to be $\mathrm{V}_{\mathrm{O}}$ and $\mathrm{O}_{\mathrm{i}}$.
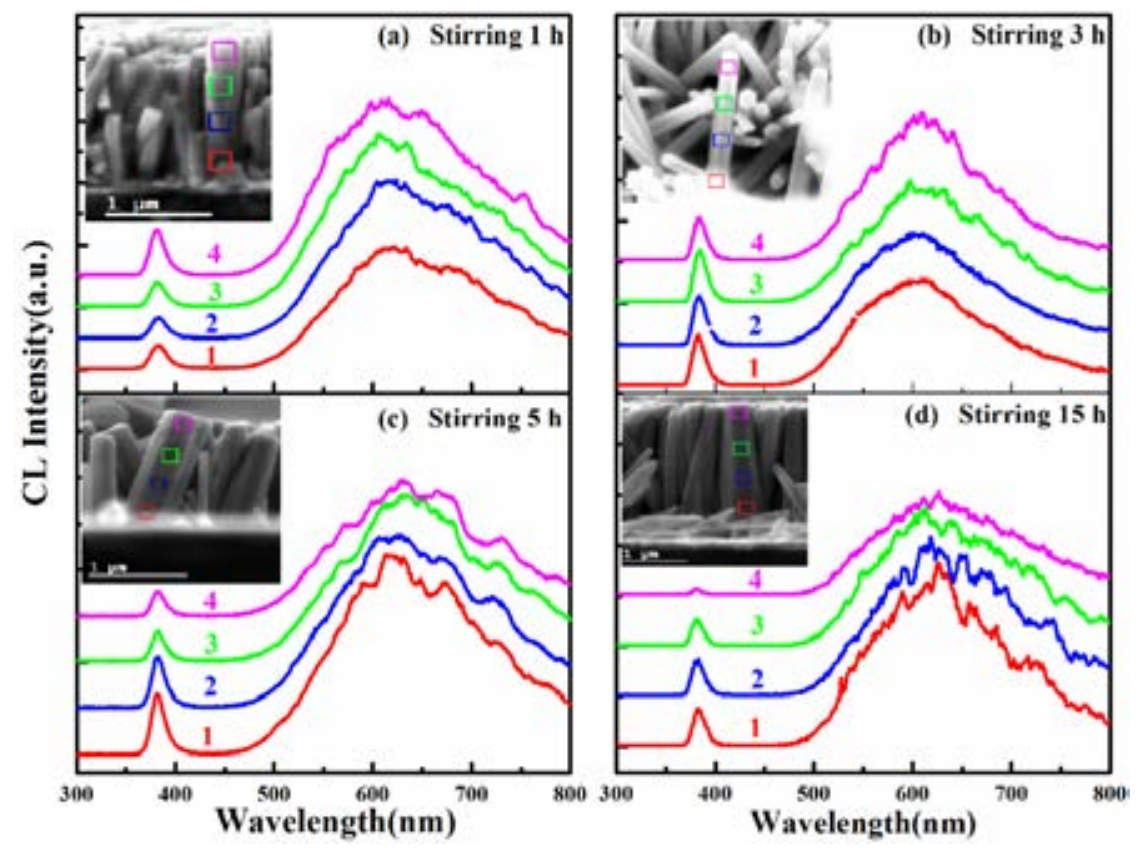

Figure 5.2: $C L$ spectra of the individual NRs measured in cross-sectional view. The insets display the cross-sectional SEM images of the NRs with a sign of the spots where the CL spectra were recorded. For clarity, the spectra have been subjected to an offset in the vertical direction. 


\subsection{Influence of the $\mathrm{ZnO}$ seed layer precursor molar ratios on the emission properties of n-ZnO NRs-based heterojunction LEDs}

$\mathrm{ZnO}$ NRs are regarded as one of the most promising candidates for the development of intrinsic white-LEDs. ${ }^{[22]}$ However, due to the lack of a method of synthesizing reproducible and stable p-type $\mathrm{ZnO}$, an alternative procedure is employed to synthesize n-type $\mathrm{ZnO}$ NRs on a p-type substrate e.g., GaN to achieve ZnO NRs-based heterojunction LEDs. ${ }^{[80-87]}$ However, the LEDs based on this configuration usually show low EL response as a result of high density of defects and different energy barriers for electrons and holes at the heterojunction interface. ${ }^{[87]}$ It is known that the interface defect states can act as non-radiative centers that will drastically diminish the efficiency of ZnO-based heterojunction LEDs. ${ }^{[85,86]}$ Previously, it has been indicated that the type of the seed layer utilized in the synthesis of the ZnO NRs can significantly modify the density of such interface defects, and so different color emissions can be observed. ${ }^{[63-65]}$ This is because the seed layer typically exists at the interfaces between the $\mathrm{p}-\mathrm{GaN}$ substrate and the synthesized $\mathrm{n}-\mathrm{ZnO}$ NRs. Consequently, manipulating the $\mathrm{ZnO}$ seed layer synthesis can be a simple and a useful path to tune the defects density at the $\mathrm{n}-\mathrm{ZnO} \mathrm{NRs} / \mathrm{p}-\mathrm{GaN}$ interfaces. In this regard, we demonstrate the possibility to tune the density of defects at the $\mathrm{n}-\mathrm{ZnO} \mathrm{NRs} / \mathrm{p}-\mathrm{GaN}$ interface by utilizing $\mathrm{ZnO}$ seed layer prepared with different molar ratios. ${ }^{[57]}$ The influence of the seed layer molar ratios on the density of defects of the synthesized $\mathrm{n}-\mathrm{ZnO} \mathrm{NRs} / \mathrm{p}-\mathrm{GaN}$ heterostructure interfaces was studied by employing spatially resolved CL spectroscopy. Interestingly, the CL results showed that the seed layer molar ratios have a notable effect on the concentration of defects at the $\mathrm{n}-\mathrm{ZnO} \mathrm{NRs} / \mathrm{p}-\mathrm{GaN}$ heterostructure interface. As can be seen, the synthesized $n-Z n O ~ N R s / p-G a N$ heterostructure obtained from a seed layer produced with a molar ratio of 
1:3 $\mathrm{M}$ as shown in Figure 5.3 (b) has a high defect emission (and consequently high defects concentrations) at the interface (red curve). ${ }^{[57]}$

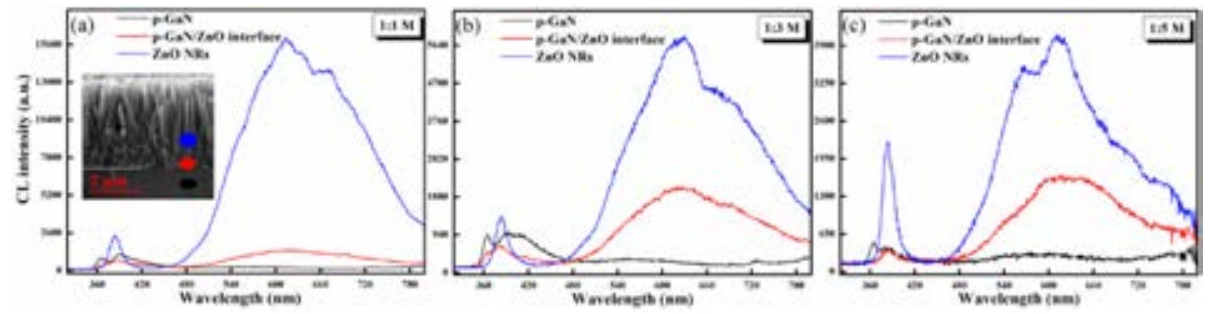

Figure 5.3: $C L$ spectra of $n-Z n O N R s / p-G a N$ heterostructure recorded in cross-sectional view. The inset displays a typical cross-sectional SEM image of the $n-Z n O N R s / p-G a N$ heterostructure with the sign of the point where the CL spectra were recorded.

Moreover, to study the impact of the seed layer molar ratios on the $\mathrm{EL}$ emission, the synthesized $\mathrm{n}-\mathrm{ZnO} \mathrm{NRs} / \mathrm{p}-\mathrm{GaN}$ heterostructures were used to fabricate LED devices. The fabricated heterojunction LEDs were marked as device 1,3 , and 5 for the $\mathrm{ZnO}$ NRs obtained using a molar ratio of 1:1, 1:3, and 1:5 M, respectively. Figure 5.4 (a)-(c) present the EL spectra from all the three LED devices monitored at varying the forward voltage/injection-currents. As can be observed, the EL spectra of all the three devices show a distinct yellow emission peak centered at $\sim 575 \mathrm{~nm}$, which can be ascribed to intrinsic DL defects emission in $\mathrm{ZnO}$ due to point defects e.g., $\mathrm{V}_{\mathrm{O}}$ and $\mathrm{O}_{\mathrm{i}}$ or be interpreted by the interface defects related emission. ${ }^{[82-85,88-91]}$ In addition to the yellow emission, a weak blue emission centered at $\sim 420 \mathrm{~nm}$ was observed for device 1 (with a 1:1 M seed layer) as presented in Figure 5.4 (a). This observation can be attributed to the transitions from the $\mathrm{CB}$ or shallow donors (in $\mathrm{ZnO}$ ) to the $\mathrm{Mg}$ acceptor levels in the $\mathrm{p}-\mathrm{GaN} .^{[83,85]}$ 

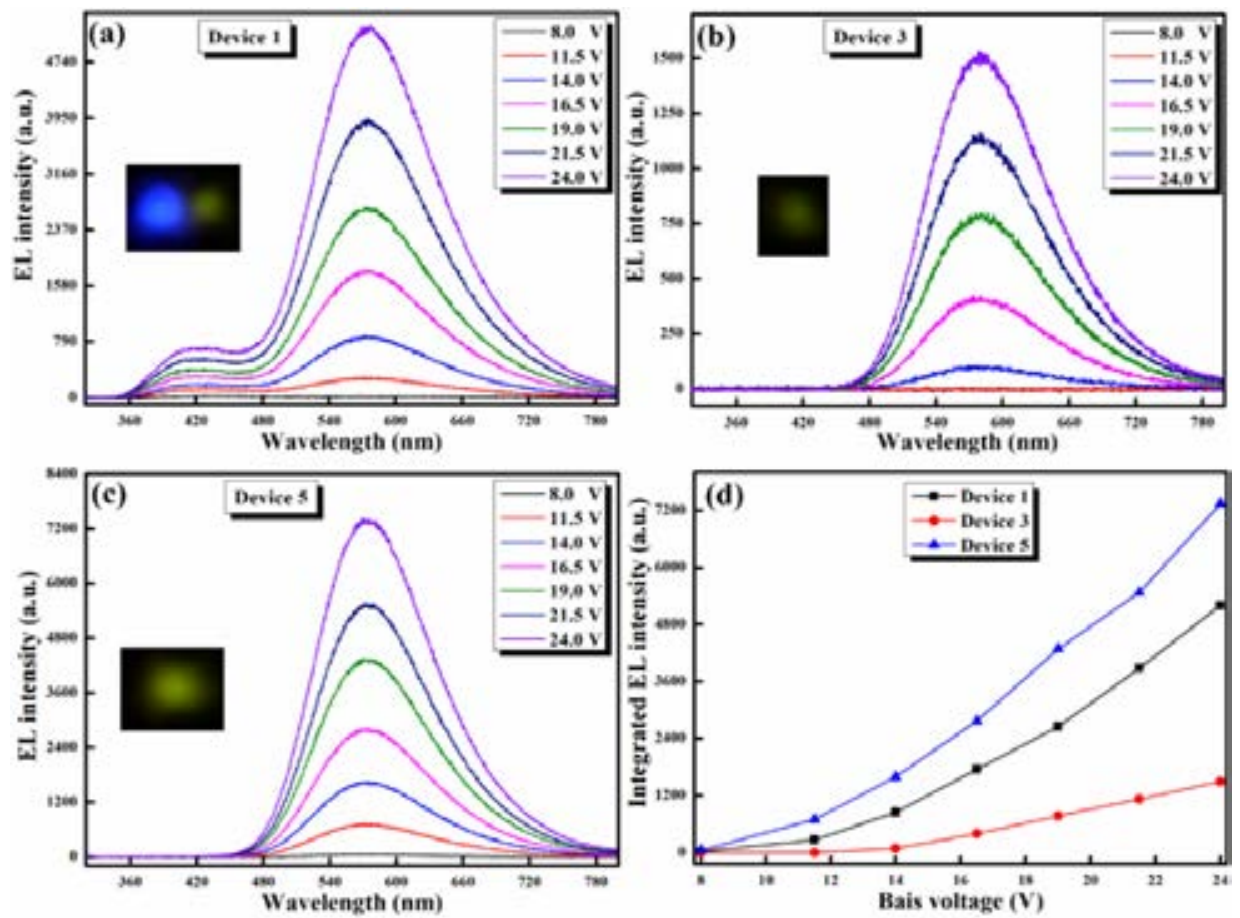

Figure 5.4: EL spectra as a function of the forward bias voltage of device (a) 1, (b) 3, and (c) 5, respectively. The insets present the corresponding light emission images at $24 \mathrm{~V}$. (d) The integrated EL intensities of the three devices as a function of the forward bias voltage.

As the bias voltage is increased, the EL intensity of the dominant emission peak for all the three LEDs increases rapidly without a notable shift in the peak position as displayed in Figure 5.4 (d). The increase in the EL intensity with increasing the bias voltage is probably due to the reduction in the band bending of the $\mathrm{n}-\mathrm{ZnO}$ NRs and the $\mathrm{p}-\mathrm{GaN}$. Therefore, the kinetic energy of the electrons and holes is increased, and they have a much higher probability to flow across the interface barrier and recombine on the opposite side of the junction. ${ }^{[85,92]}$ 
Furthermore, the explanation of the mechanism of the enhanced EL intensity of the yellow emission from the fabricated heterostructure LEDs is given below. The integrated EL peak intensity as a function of the injection current is plotted on a log-log scale as shown in Figure 5.5 for all the three LEDs. The results can be fitted with the law $L=c I^{m}$, where $m$ measures the impact of the non-radiative defects in the characteristics of the light emission. A linear rise in $L$ with $I$ e.g., $m \sim 1$ can be assumed when radiative recombination dominates, while non-radiative recombinations become dominant when $L$ displays a super-linear dependence on the $I$ e.g., when $m>1 .{ }^{[93,94]}$ Our result revealed a super-linear dependence with $m=$ 2.3, 4.0, and 1.9 for devices 1,3 , and 5 , respectively. These values indicate the role of the defect-related non-radiative recombination centers for all the three LEDs, which is in good agreement with the CL spectra in Figure 5.3. However, the value of $m$ is defined from the fitting curve for device 5 (with $1: 5 \mathrm{M})$ is the lowest $(m=1.9)$, which shows a low presence of non-radiative recombination centers in the space-charge region. Since the low density of the interface defects is favorable for improving carrier injection through the heterojunction, device 5 shows an improved EL performance compared to the other two devices 1 and 3 . These results suggest that the molar ratios of the $\mathrm{ZnO}$ seed layer have a significant effect on the density of interface defects and, in particular, playing a role of non-radiative recombination centers. 


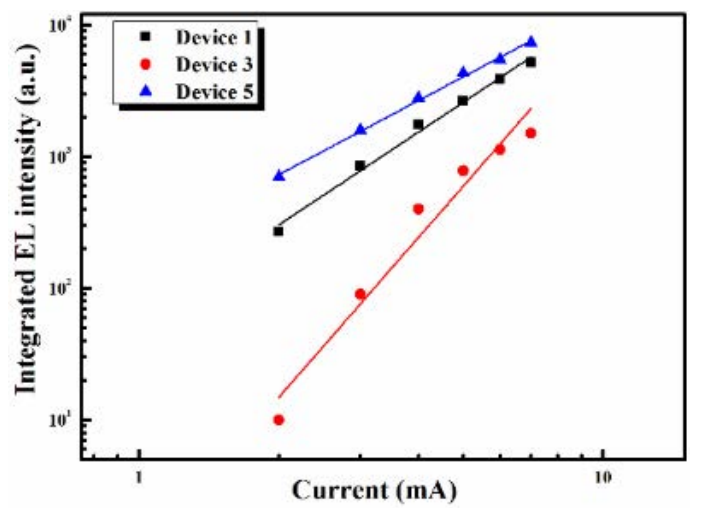

Figure 5.5: Integrated EL peak intensities of all the three devices as a function of the forward injection current. The solid lines represent the fitting results based on the power law $L=c I^{m}$.

\subsection{Influence of $\mathrm{ZnO}$ seed layer precursor types on the emission properties of n-ZnO NRs-based heterojunction LEDs}

Proceeding with the investigation of the effect of the $\mathrm{ZnO}$ seed layer on the emission properties of $\mathrm{n}-\mathrm{ZnO}$ NRs-based heterojunction LEDs and by choosing the best-optimized seed layer molar ratio. We further studied the influence of the seed layer precursor types on the emission properties of $\mathrm{n}-\mathrm{ZnO}$ NRs-based heterojunction LEDs. ${ }^{[58]}$ Figure 5.6 (a) displays the top-view $\mathrm{CL}$ spectra of the synthesized $\mathrm{n}-\mathrm{ZnO}$ NRs/p-GaN heterostructures, which is described by a weak NBE emission peak centered at $\sim 383 \mathrm{~nm}$ and a dominant a yellow-orange peak centered at $\sim$ $610 \mathrm{~nm}$ related to DL defects emission. ${ }^{[82,85,88-91]}$ The yellow-orange emission is usually observed in the $\mathrm{ZnO}$ NRs synthesized by the LT-ACS, and it is stated to be due to DL defects emission e.g., $\mathrm{V}_{\mathrm{O}}$ and $\mathrm{O}_{\mathrm{i}}$ in $\mathrm{ZnO}$ or can be caused by the $\mathrm{V}_{\mathrm{Zn}}$ and hydrogen complex defects. ${ }^{[20]}$ As can be observed from Figure 5.6 (a) the synthesized n-ZnO NRs/p-GaN NRs heterostructure obtained from a seed layer prepared from $\mathrm{ZnAc}, \mathrm{KOH}$ and 
HMTA (donated as ZKH seed) displayed good crystal quality and low defects concentrations compared to that prepared from $\mathrm{ZnAc}$ and $\mathrm{KOH}$ (donated as ZK seed), as deduced from the ratio of the NBE to DL defect emission. ${ }^{[63]}$ Moreover, as revealed by spatially resolved $\mathrm{CL}$, the synthesized n-ZnO NRs/ p-GaN heterostructure using the ZKH seed layer shows a significant reduction in the interface defects as illustrated in figure 5.6 (b). This decrease in the CL intensity of the defects emission is likely to be due to the different defects concentrations at the interface between the two types of the $\mathrm{ZnO} \mathrm{NRs} / \mathrm{p}-\mathrm{GaN}$ heterostructures e.g., higher for $\mathrm{ZnO}$ NRs achieved using the ZK seed solution. ${ }^{[57]}$
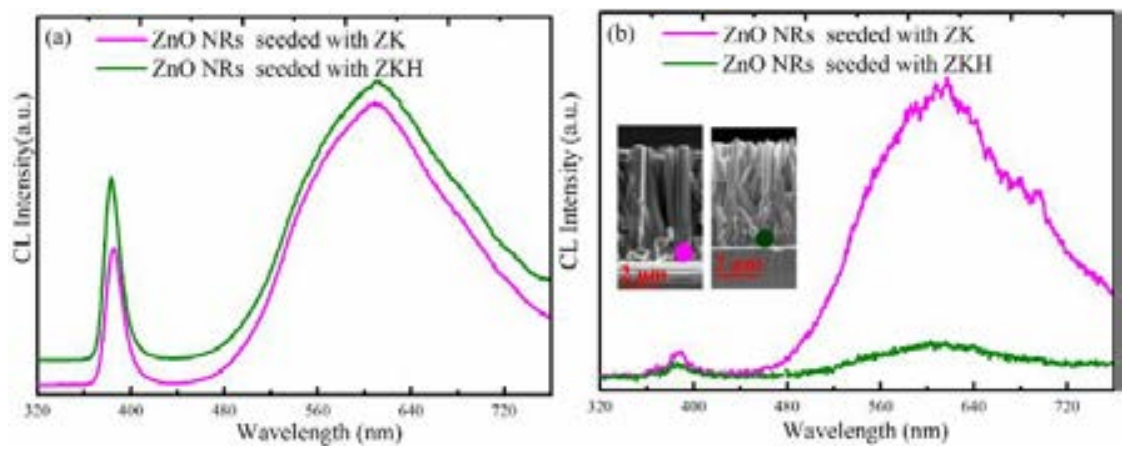

Figure 5.6: (a) CL spectra of the $n-Z n O N R s / p-G a N$ heterostructures recorded in top-view mode. For clarity, all the spectra are normalized to the $D L$ defects emission and shifted in the vertical direction. (b) Crosssectional view $C L$ spectra of the $n-Z n O N R s / p-G a N$ heterostructures interface. The insets display the synthesized $n-Z n O \quad N R s / p-G a N$ heterostructures in cross-sectional view with a sign of the points where the CL spectra were measured.

Further, the EL of the fabricated LED devices was performed at different bias voltages to understand the evolution and tuning of the emission through the $\mathrm{ZnO}$ seed layer precursors, as displayed in Figure 5.7 
(a)-(b). The EL intensity of the dominant emission peak increases rapidly without a notable shift in the peak position with increasing the bias voltage. The dominant broad yellow emission has a slightly asymmetric shape and therefore can consists of several DL defects emission transitions levels. Figure 5.7 (c) displays an example of the peak decomposition applying a Gaussian function for the experimental spectrum performed at a bias voltage of $15 \mathrm{~V}$ for the $\mathrm{ZKH}$ device, which shows that the dominant broad emission peak consists of three separate visible defect emission bands centered at $\sim 551,597$, and $652 \mathrm{~nm}$, respectively. These visible defects emission is probably due to transitions from the CB to DL defects emission induced by $\mathrm{V}_{\mathrm{O}}$ and $\mathrm{O}_{\mathrm{i}}$ in the $\mathrm{ZnO}{ }^{[57,82,85,94]}$ or could be due to radiative interface recombinations within the $\mathrm{n}-\mathrm{ZnO} \mathrm{NRs}$ and the $\mathrm{p}-\mathrm{GaN}$ substrate ${ }^{[63,64]}$ The difference in the emission spectra of the ZH device and the ZHK device in Figure 5.7 (d) can be explained by the difference in the density of interface point defects as indicated in Figure 5.6 (b). Consequently, the band alignment in the $\mathrm{n}-\mathrm{ZnO} \mathrm{NRs} / \mathrm{p}-\mathrm{GaN}$ is predicted to be different, and hence a variation in carrier recombination can be observed. ${ }^{[63]}$

An apparent increase in the EL intensity of the dominant emission peak for the two LEDs is seen as the bias voltage is increased as displayed in Figure 5.8 (a). The improvement in the EL intensity with increasing the bias voltage is probably due to the lowering of the band bending of the n$\mathrm{ZnO} \mathrm{NRs}$ and the $\mathrm{p}-\mathrm{GaN}{ }^{[85,93]}$. As a result, the kinetic energy of the electrons and holes is increased, and they have a much higher chance to go across the interface barrier and recombine on the opposite the side of the heterojunction. ${ }^{[82,85,93]}$ 

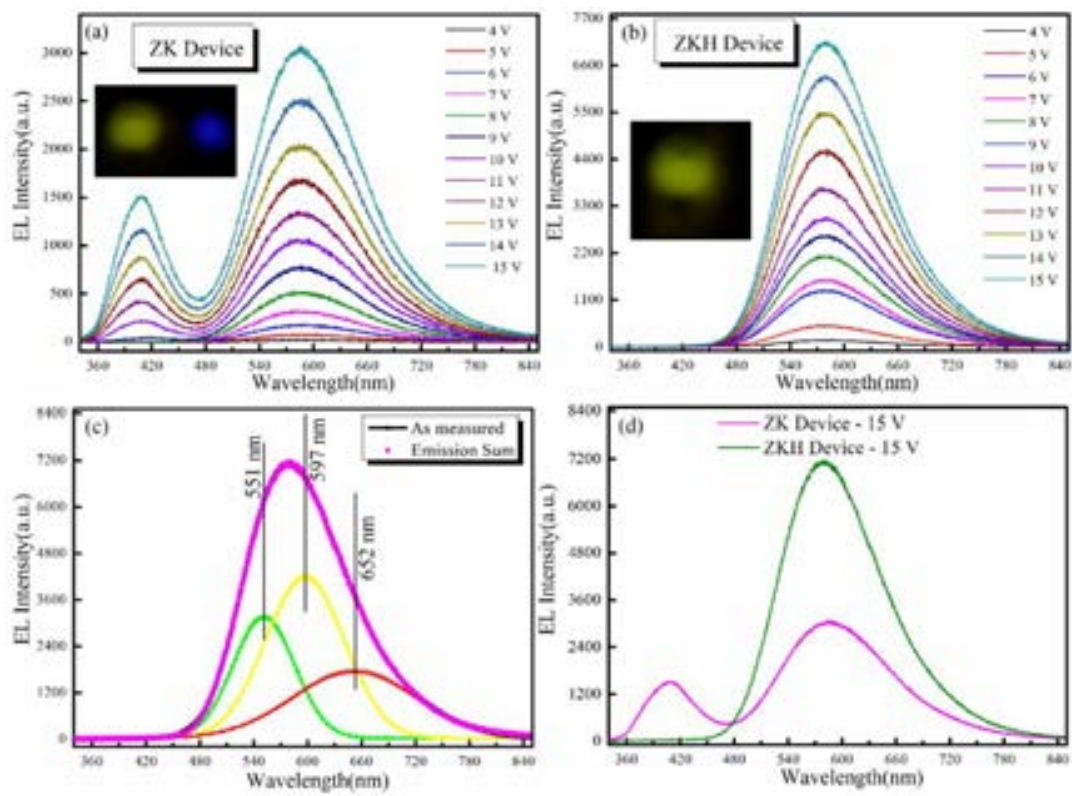

Figure 5.7: EL spectra of (a) ZK device, and (b) ZKH device, as a function of the bias voltage. The insets show the corresponding light emission images at $15 \mathrm{~V}$. (c) Gaussian decomposition of the dominant yellow emission of the ZKH device at a bias voltage of $15 \mathrm{~V}$. (d) EL spectra of the two devices under a bias voltage of $15 \mathrm{~V}$.

Further, the integrated intensity of the EL peak as a function of the injection current is plotted on a log-log scale as shown in Figure 5.8 (b) to illustrate the mechanism of the improved EL from the fabricated ZKH LEDs. The curve fitting results exhibited a super-linear dependence with $n$ $=2.0$ for the $\mathrm{ZK}$ and 1.3 for the $\mathrm{ZKH}$ device, respectively (as deduced from

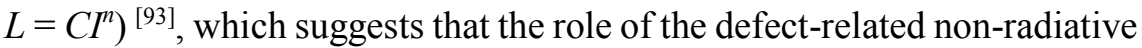
recombination centers is dominant for all the two LEDs. However, the value of $n$ (1.3) for the ZKH device indicates a comparatively lower existence of non-radiative recombination centers in the space-charge region. Since the low concentration of interface defects is convenient and 
is a benefit for carrier injection through the heterojunction. The ZKH device displayed an enhanced EL performance in contrast to the ZK device as shown in Figure 5.7 (d). These results imply that the $\mathrm{ZnO}$ seed layer precursor has a significant effect on the interface defect properties and precisely it is playing an important role in the concentration of the nonradiative recombination centers.

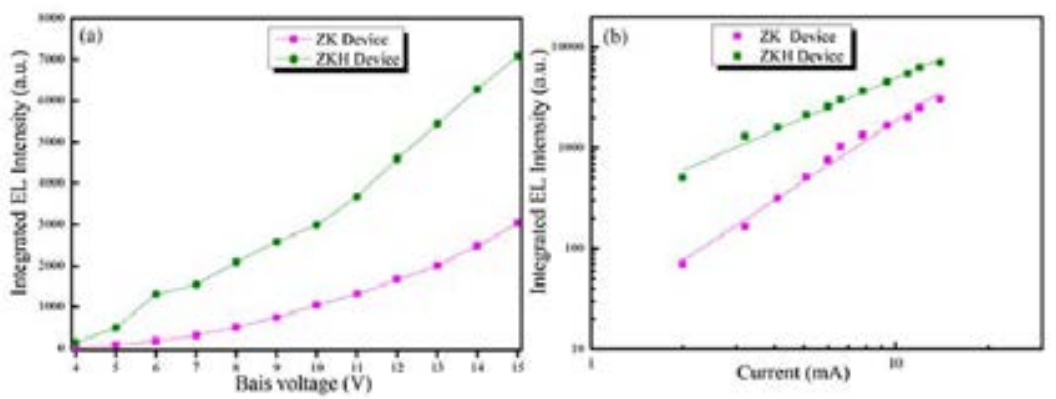

Figure 5.8: (a) The integrated EL peak intensities of the $Z H$ and $Z K H$ devices as a function of the bias voltage, and (b) Role of injection current. The solid lines in Figure b illustrate the fitting results based on the power law $L=C I^{n}$.

\subsection{Synthesis of Co-doped $\mathrm{ZnO}$ oriented NRs}

Doping is a prime method to tune the semiconductor properties e.g., optical, electronic and magnetic. Recently, theoretical predictions and experimental research have established that the incorporation of TMs e.g., iron $(\mathrm{Fe})$, nickel $(\mathrm{Ni})$, manganese $(\mathrm{Mn})$, cobalt $(\mathrm{Co})$, and copper $(\mathrm{Cu})$ in $\mathrm{ZnO}$ will result in a potential for the development of RT-FM. ${ }^{[47-50]}$ Among a variety of TMs-doped $\mathrm{ZnO}$, Co is of particular interest because it has unique optical and magnetic properties. ${ }^{[51,52,95-98]}$ Consequently, substantial research efforts have been devoted to synthesize Co-doped $\mathrm{ZnO}$ utilizing different synthesis methods. Most of this research has been concentrated 
on investigating the optical and magnetic properties of Co-doped $\mathrm{ZnO} .^{[48-}$ ${ }^{51]}$ However, the synthesis of a high crystal quality of Co-doped $\mathrm{ZnO}$ with the successful substitution of $\mathrm{Co}^{2+}$ in the host crystal site is still challenging. The successful realization of high crystal quality Co-doped $\mathrm{ZnO}$ will probably lead to better understanding of the origin of the RT-FM in $\mathrm{ZnO}$. In particular, doping of $\mathrm{Co}$ ions in $\mathrm{ZnO}$ crystal lattice utilizing the low-temperature solution process can be challenging because the dopant species can easily create aquo ions and not incorporated in the crystal lattice. ${ }^{[39]}$ Here, by developing a process for preparing the synthesis solution, we synthesized well-oriented Co-doped $\mathrm{ZnO} \mathrm{NRs}$, and the crystal structure was studied at low-temperature using EPR. ${ }^{[52]}$ Figure 5.9 presents a top-view SEM images of the synthesized pure (S0) and 5\% Co-doped ZnO NRs (S2) samples, which have a characteristic length of $3.5 \mu \mathrm{m}$ and mean diameters of 220 and $300 \mathrm{~nm}$, respectively.
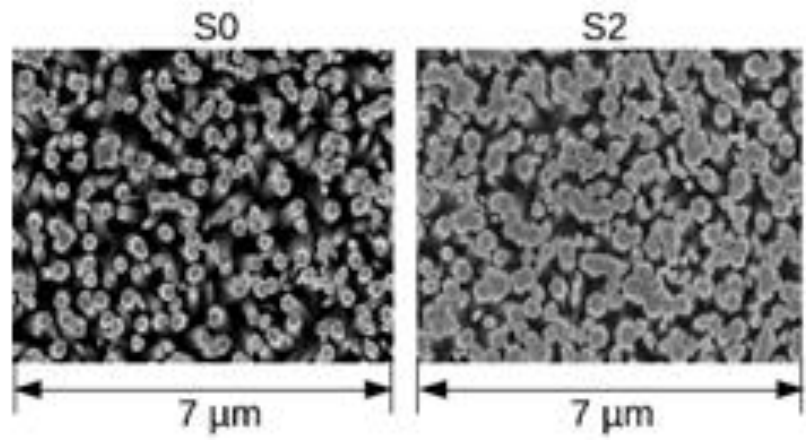

Figure 5.9: Top-view SEM images of the pure (SO) and 5\% Co-doped $\mathrm{ZnO} N \mathrm{Ns}$ (S2), respectively.

The EPR spectra of the pure sample (S0) are defined by the wellknown defect signal from $\mathrm{ZnO}$ apparent at $350 \mathrm{mT}(\mathrm{g} \sim 1.956)$ as displayed in Figure 5.10, and usually, assigned to core-defects (CDs) resulting from $\mathrm{ZnO}$ NSs rather than shell defects. ${ }^{[99-101]}$ This $\mathrm{g}(\sim 1.96)$ CD signal is 
completely resolved by lowering the static magnetic $(\boldsymbol{B})$ field modulation from 5 to $1 \mathrm{G}$, then losing intensity in the same ratio, but letting a threeline structure appears (Figure 5.11), which implies the presence of three different and independent two-levels resonant centers, rather than a multilevels one. Nevertheless, we do not intend to discuss the defect's nature; here we simply model the experimental data by a set of three independent $1 / 2$ spins, with axially anisotropic g-factors: this is a mean of recording the experimental facts (relative transition intensities, line position, and width), for later comparison or modeling.

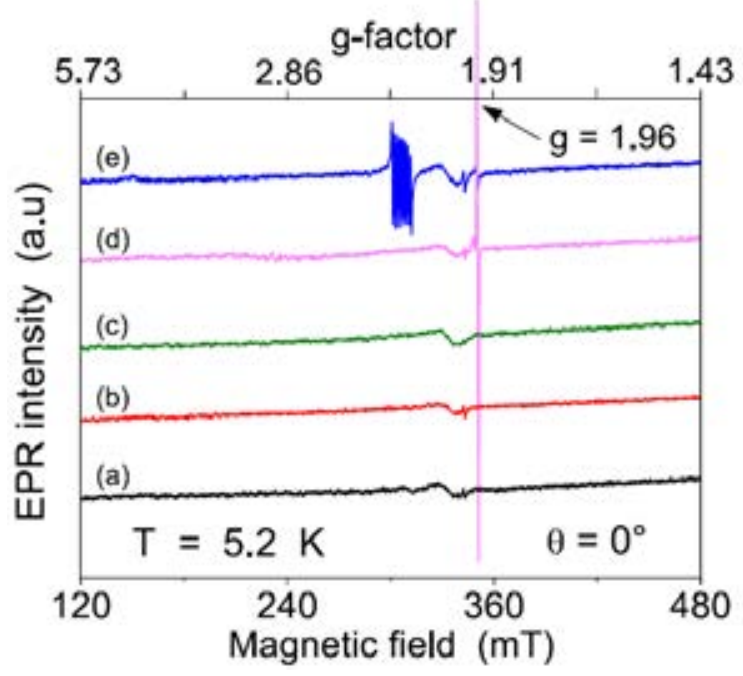

Figure 5.10: $X$-band EPR spectra (5 G modulation) at each step of the NRs synthesis. (a) The sample holder, (b) Sapphire substrate, (c) ZnO seed layer, (d) Pure NRs, and (e) 5\% of Co-doped ZnO NRs. No baseline subtraction or exponential smoothing.

In performing so, a reasonable fit of the angle-dependent spectra for Figure 5.11 showed that one of these defect's line is almost isotropic $\left(D_{3}, g_{\|} \cong \mathrm{g}_{\perp}\right.$, $\mathrm{g}_{\|}=1.9602$ and $\left.\sim \mathrm{g}_{\perp}=1.9604\right)$ ), while the other two $\left(\mathrm{D}_{1}, \mathrm{~g}_{\|}=1.9647, \mathrm{~g}_{\perp}\right.$ 
$=1.9630$, and $\mathrm{D}_{2}, \mathrm{~g}_{\|}=1.9619, \mathrm{~g}_{\perp}=1.9607$ ) display a slight axial anisotropy, in consistency with the hexagonal wurtzite structure of the $\mathrm{ZnO}$ NRs. ${ }^{[52]}$ The observation of this anisotropy is a first evidence that the NRs are single crystalline and are mostly vertically oriented. We summarize the pure NRs under study by noting that their EPR signals are well identified. They contain known $\mathrm{ZnO}$ CDs and were mostly aligned perpendicularly to the substrate surface, and no detectable traces of defects or impurities are observed, a fact which indicates the relatively high purity of these samples.

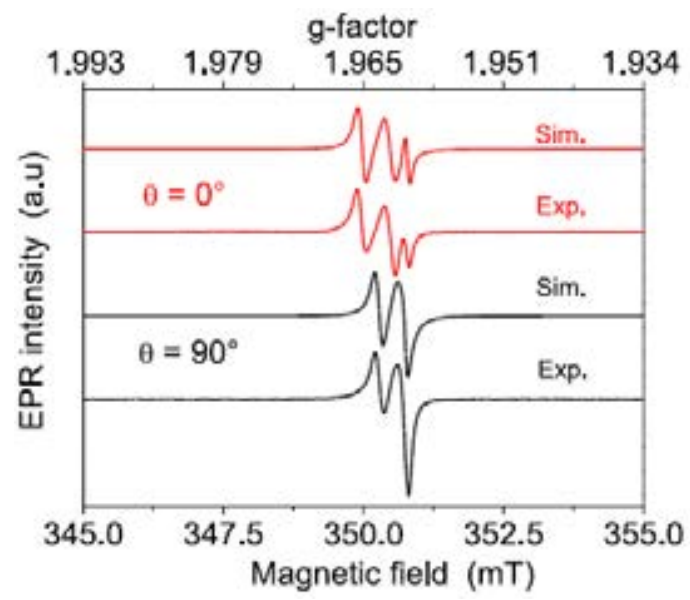

Figure 5.11: Experimental $X$-band EPR spectra ( $1 G$ modulation) of sample SO showing the $g \sim 1.96$ signal for $B / / z\left(\theta=0^{\circ}\right)$ and $B \perp z(\theta=$ $90^{\circ}$ ), recorded at $T=5 \mathrm{~K}$. The upper $x$-axis gives the corresponding $g$ factor value.

The EPR spectra of the 5\% Co-doped NRs (samples S2), is significantly characterized by a reduction of the CDs signal $(g \sim 1.96)$ intensity which is observed in S0 ( Figure 5.10) and the appearance of a Co-related signal at $\mathrm{g} \sim 2.24$, for $\theta=0^{\circ}$, and at $\mathrm{g} \sim 4.58$ for $\theta=90^{\circ}$ as displayed in Figure 5.12. The observed reduction of the $\mathrm{CD}$ is an interesting sign that a certain amount of the incorporated Co is involved in 
the neutralization of the CDs. Whereas the observed magnetic anisotropy of the $\mathrm{Co}^{2+}$ signal is a clear evidence that the NRs are single crystalline and are vertically well aligned, at least everywhere the Co ions supply an EPR signal. ${ }^{[52]}$

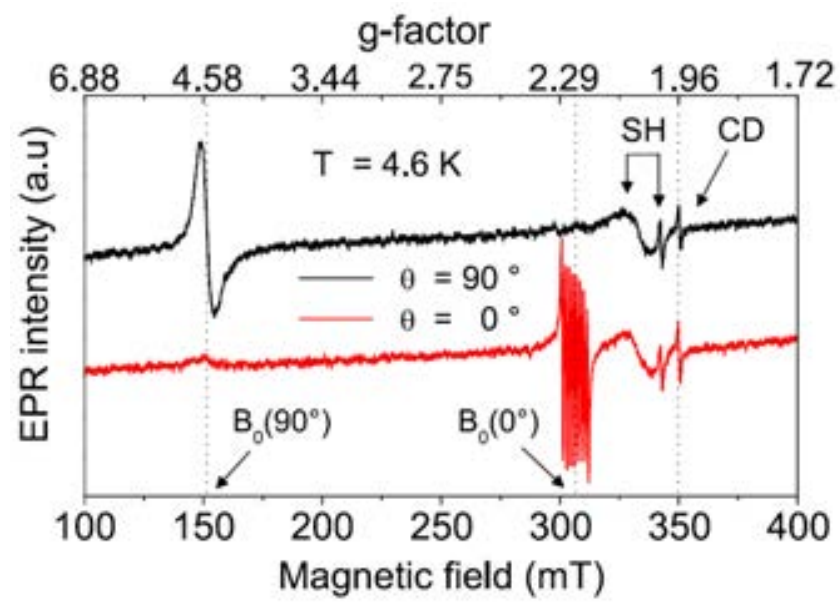

Figure 5.12: $X$-band EPR spectra of sample $S 2$ for parallel $\left(\theta=0^{\circ}\right)$ and perpendicular $\left(\theta=90^{\circ}\right)$ orientation of the $B$ field. The $C D$ and sample holder (SH) signals are indicated. Gravity centers of the $\mathrm{Co}^{2+}$ signal for both orientations are indicated.

\subsection{The influence of the synthesis solution mixing on Co-doped $\mathrm{ZnO}$ NRs}

To confirm our approach for the synthesis of high crystal quality Co-doped $\mathrm{ZnO}$ NRs using the LT-ACS route, we further examined the effect of the mixing the synthesis solution on the substitution of Co ions in the $\mathrm{ZnO}$ crystal lattice while preserving the NRs morphology and alignment. In general, the synthesis of Co-doped $\mathrm{ZnO}$ NRs using the LTACS route is carried out by adding the Co ions source to the already prepared mixture of the $\mathrm{Zn}$ salt with HMTA synthesis solution. Here, in 
addition to the above method for the synthesis of Co-doped ZnO NRs, we adapted another way by mixing the HMTA with Co dopant source first and then adding the $\mathrm{Zn}$ salt. ${ }^{[34,52,59]}$

Figure 5.13 displays a top-view FE-SEM images of the synthesized pure (M0) and Co-doped ZnO NRs synthesized by mixing Zn salt with HMTA and then adding Co salt ( labeled as M1), mixing Co salt with HMTA and then adding Zn salt (labeled as M2). Vertically aligned NRs with a hexagonal shape and having an average diameter of $\sim 160, \sim 400$, and $\sim 200 \mathrm{~nm}$ for M0 (pure ZnO NRs), M1, and M2, respectively, were obtained as shown in Figure 5.13. The notable and slight increase in the NRs average diameter between the M1 and M2 as compared to M0 is possibly due to the Co doping. ${ }^{[102,103]}$

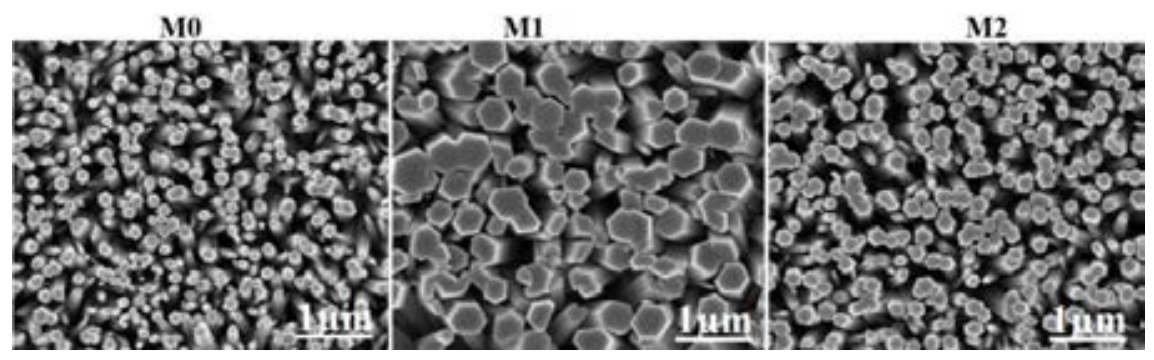

Figure 5.13: Top-view FE-SEM images of pure (MO) and Co-doped $\mathrm{ZnO}$ NRs synthesized using approaches $M 1$ and $M 2$, respectively.

The crystal structure quality of the synthesized pure and Co-doped ZnO NRs has been confirmed by the XRD measurements as illustrated in Figure 5.14. The XRD patterns showed that all the synthesized samples had a wurtzite structure with a good crystal quality and preferred growth orientation along the c-axis, as indicated by the intensity of the (002) peak. No secondary phase related to Co was seen in the XRD patterns of the pure and Co-doped $\mathrm{ZnO}$ NRs samples. The slight shift in the (002) peak position 
toward lower $2 \theta$ angle in M1 and higher $2 \theta$ angle in M2 (as shown in the inset of Figure 5.14) as compared to M0 is an indication of the successful incorporation of the Co or the due to the difference of defects density in the synthesized NRs. ${ }^{[103-105]}$

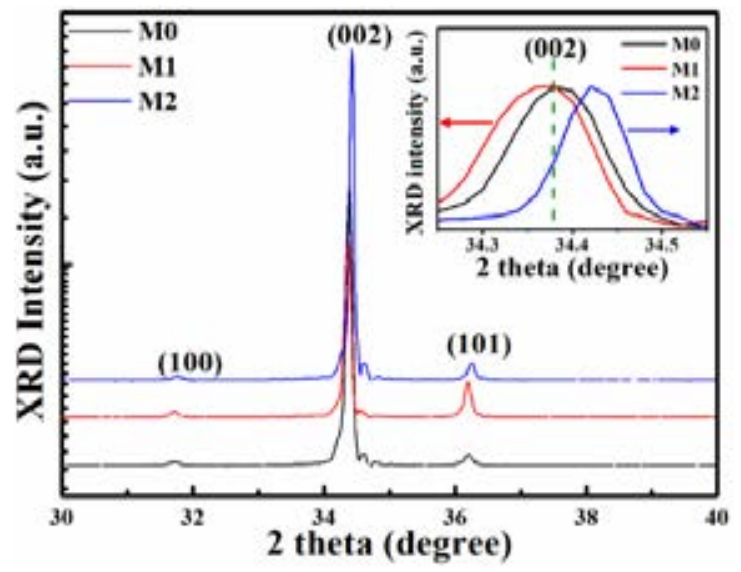

Figure 5.14: XRD patterns of the synthesized pure (MO) and Co-doped $\mathrm{ZnO} N \mathrm{Rs}$ (M1 and M2). The inset shows the normalized XRD data for the (002) peaks, indicating peaks shift.

The effect of the incorporation of $\mathrm{Co}$ into the $\mathrm{ZnO}$ using the two different methods (M1 and M2) was investigated using EPR measurements. The successful substitution of $\mathrm{Co}^{2+}$ was confirmed by the observed Co-related signal defined by an eight-line structure at $\mathrm{g} \sim 2.239$ $\left(\theta=0^{\circ}\right)$ and a broad asymmetric signal at $\mathrm{g} \sim 4.517\left(\theta=90^{\circ}\right)^{[51,52]}$ as displayed in Figure 5.15. The detected magnetic anisotropy of the $\mathrm{Co}^{2+}$ signal is clear evidence that the synthesized $\mathrm{ZnO}$ NRs are single crystalline, well-aligned, and that Co is highly diluted along the NRs. ${ }^{[36]}$

As presented in Figure 5.15, the intensity of the $\mathrm{Co}^{2+}$ signal of M2 at $\theta=90^{\circ}$ and $\theta=0^{\circ}$ is significantly higher as compared to that of M1. Also, the line width of the $\mathrm{Co}^{2+}$ signal at $\theta=0^{\circ}$ for $\mathrm{M} 2$ is observed to be 
slightly smaller (4G) than that of M1 (5G). As the Co concentration in the synthesis solution is the same (7\%) for both M1 and M2, and by considering a uniform doping and the same coverage of the NRs, these results reveal that the way of preparing the synthesis solution has a significant influence on the Co incorporation in the synthesized $\mathrm{ZnO}$ NRs in agreement with the XRD results displayed in Figure 5.14.

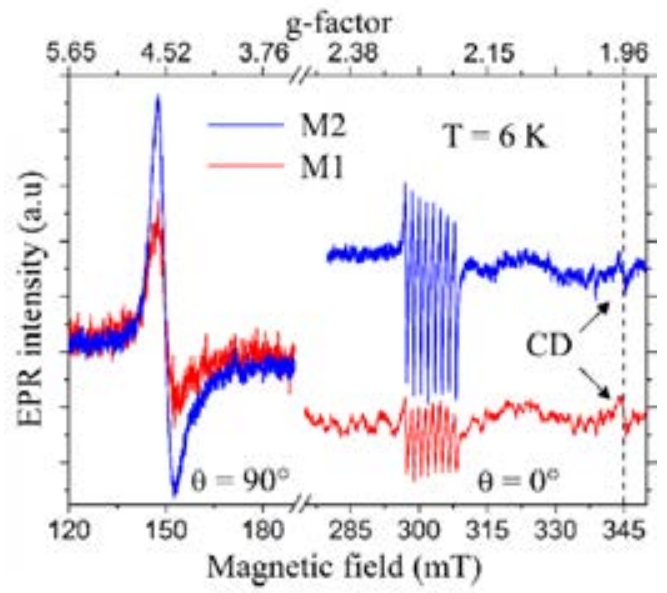

Figure 5.15: EPR spectra of Co-doped $\mathrm{ZnO} N R s$ (M1 and M2) for parallel $\left(\theta=0^{\circ}\right)$ and perpendicular $\left(\theta=90^{\circ}\right)$ orientation of the magnetic field, recorded at $T=5 \mathrm{~K}$.

Moreover, to get more information on the defects in the synthesized pure and Co-doped $\mathrm{ZnO} \mathrm{NRs}$, measurement and analysis of the roomtemperature CL spectra were carried out, and the results are presented in Figure 5.16. The emission spectra of all samples were dominated by a UV emission peak centered at $\sim 382 \mathrm{~nm}(3.24 \mathrm{eV})$ due to NBE emission and strong broad yellow-orange emission centered at $\sim 610 \mathrm{~nm}(2.03 \mathrm{eV})$ connected with the DL defects associated emissions in $\mathrm{ZnO}{ }^{[46,105]}$ Apparently, the CL spectra of the Co-doped ZnO NRs displayed a small red-shift of the UV peak position from $382 \mathrm{~nm}$ to $384 \mathrm{~nm}$ (as displayed in 
the inset of Figure 5.16) as compared to pure $\mathrm{ZnO}$ NRs, which is likely to be due to the change in band structure as a result of Co doping. ${ }^{[102,105]}$ It is important to note that the $\mathrm{CL}$ defect-related yellow-orange emission intensity decrease from M1 to M2 (Figure. 5.16), and the Co EPR signal increases from M1 to M2 (Figure 5.15). These observations propose that the method of preparing the synthesis solution has a substantial impact on the Co incorporation and defect generation in the synthesized $\mathrm{ZnO}$ NRs in agreement with the XRD results shown in Figure 5.14. The physical source of intrinsic defects-related yellow-orange emission is doubtful, and it is stated to be linked to the $\mathrm{Vo}, \mathrm{O}_{\mathrm{i}}$, and $\mathrm{Zn}_{\mathrm{i}}$. Recently, it was suggested that the defect-related orange emission is to possibly be originating due to the $\mathrm{Zn}_{\mathrm{i}}$ in the core of $\mathrm{ZnO}$ NRs. ${ }^{[106]}$

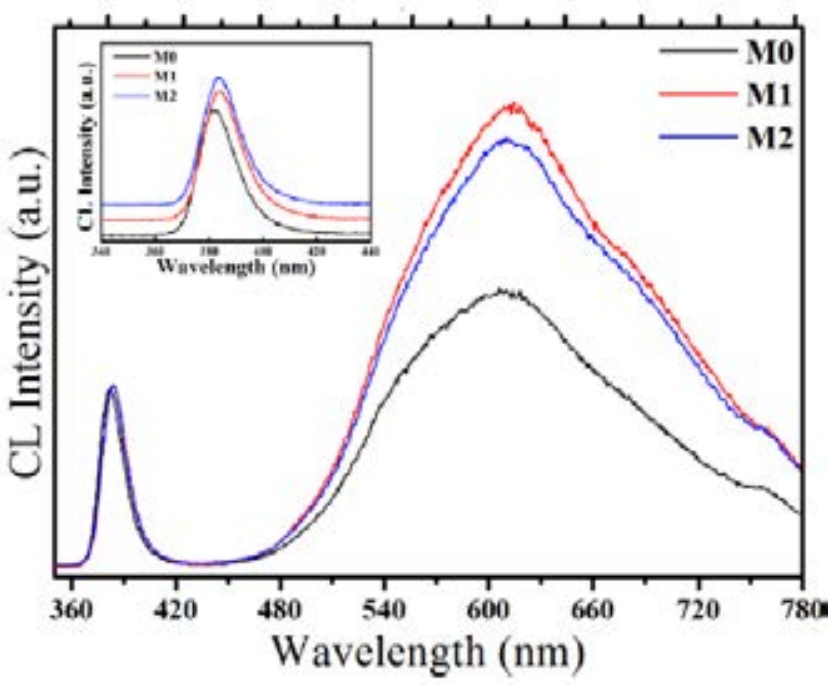

Figure 5.16: $C L$ spectra of the synthesized pure and Co-doped $\mathrm{ZnO} N R s$ obtained by utilizing different synthesis preparation approaches as indicated. The inset demonstrates the red-shift in the UV peak. For clarify, the spectra are normalized to the NBE intensity. 
In this study, we suggest that the defect-related yellow-orange emission is likely to originate from $\mathrm{Zn}_{\mathrm{i}}$ in the core of $\mathrm{ZnO}$ NRs or from the $\mathrm{Vo}$ and $\mathrm{O}_{\mathrm{i}}$ on the surface of $\mathrm{ZnO}$ NRs. As a result, the intensity of the defect-related yellow-orange emission is significantly improved by the Codoping (Figure 5.16), which is presumably due to the increase in Vo and $\mathrm{O}_{\mathrm{i}}$ in the NRs or to the Co-related defect. ${ }^{[15,23,41]}$ This suggests that the above-observed red-shift of the UV peak could be ascribed to the variation of the $\mathrm{Zn}_{\mathrm{i}}$ concentrations in the Co-doped samples (M1 and M2) compared with the pure $\mathrm{ZnO}$ NRs (M0). In fact, these results indicate that the bulk quality of the $\mathrm{ZnO}$ NRs is improved by the substitution of $\mathrm{Co}^{2+}$, while the doping has the adverse effect on the surface defects-related emission, in agreement with early results. ${ }^{[105,107]}$

In view of the EPR and the CL results, a defect distribution model for $\mathrm{ZnO}$ NRs is proposed as shown in Figure 5.17 $7^{[108]}$, which states that the incorporation of $\mathrm{Co}^{2+}$ during the synthesis process could reasonably result in filling $\mathrm{Zn}_{\mathrm{i}}^{+}$by substitutional or interstitial doping and, consequently, improved the crystal quality of the synthesized ZnO NRs. ${ }^{[51]}$ The other possibility is that a substitutional $\mathrm{Co}^{2+}$ very close to a $\mathrm{Zn}_{\mathrm{i}}^{+}$may form a nonmagnetic complex, which is then not anymore EPR detectable.

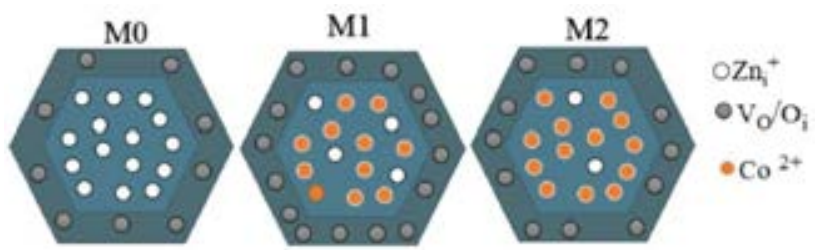

Figure 5.17: Schematic diagram of the cross-sectional view of the synthesized pure and Co-doped $\mathrm{ZnO}$ NRs including $\mathrm{Zn}_{i}{ }^{+}$as core-defect and oxygen vacancies/interstitials as surface defects, respectively. 
Moreover, the incorporation of $\mathrm{Co}^{2+}$ was observed to lead to the increased concentration of surface defects such as $\mathrm{V}_{O}$ and $\mathrm{O}_{i}$. However, further experimental investigation coupled with comprehensive theoretical calculations are required to fully understand this observed phenomena.

\subsection{Core-defect reduction in ZnO NRs by Co incorporation}

As mentioned previously, $\mathrm{ZnO}$ NRs have a potential for the development of a diverse range of functional electronic devices e.g., LEDs ${ }^{[22]}$ and UV detectors. ${ }^{[25-27]}$ In these applications, intrinsic and extrinsic defects play a significant role in their optical and electrical properties. Consequently, information about and control of these defects, as well as the relation between them, are essential factors for the optimization of the electronic and optical properties of $\mathrm{ZnO}$ NRs. In particular, the paramagnetic defects are of interest because when an externally applied magnetic field, the majority of electrons (depending on temperature) have up (or down) spin projection, which then restricts the possible recombination paths via these polarized defects to those allowed by Pauli exclusion principle. Regarding these paramagnetic defects, the EPR is a valuable tool to characterize them and to examine their occurrence as a function of the different synthesis parameters. ${ }^{[34,52,77]}$ Simultaneously, the EPR spectra can give information about the spin environment, the crystal quality at the atomic scale, and the macroscopic ordering. However, applying the EPR spectroscopy on paramagnetic NSs is tricky, mainly due to the moderate amount of resonating centers and the large spin relaxation. Moreover, as most of the previous studies deal with non-oriented NPs, information on magnetic anisotropy is often lost. Therefore, here we synthesized well-oriented pure and Co-doped ZnO NRs by the LT-ACS $\left(90{ }^{\circ} \mathrm{C}\right)$ with different Co concentrations within the synthesis solution 
(from $0 \%$ to $15 \%$ ), and are investigated by the EPR, just above the liquid helium temperature. The analysis of the EPR spectra shows that a decrease in the intensity of the paramagnetic native $\mathrm{CD}$ at $\mathrm{g} \sim 1.96$ with the increase of Co in the synthesis solution, proposing a gradual neutralization of the former by the latter as presented in Figure 5.18. However, the comparison of absolute intensity in different samples does not lead to significant information since the total number of the NRs may vary from a sample to another, mainly due to the differences in the substrate coverage.

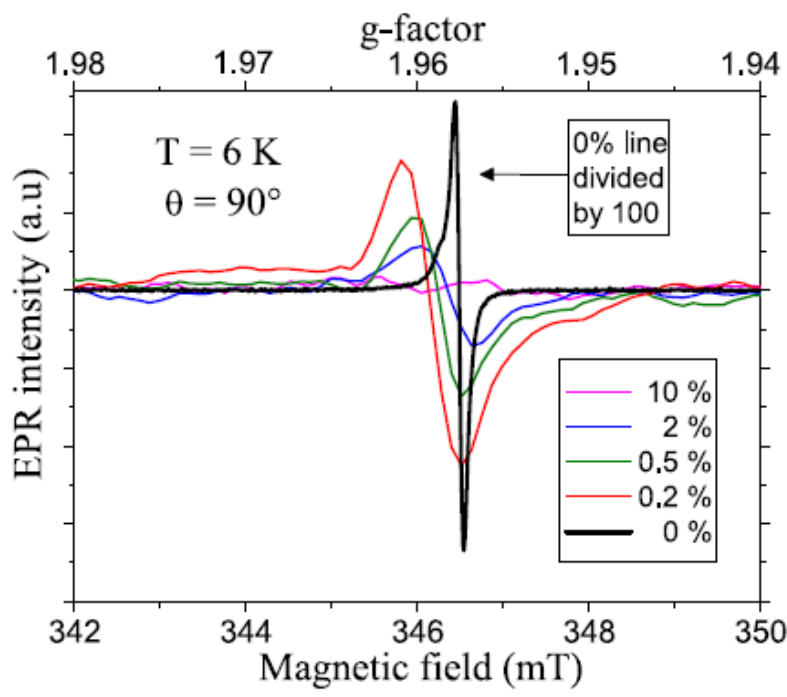

Figure 5.18: Evolution of the CDs signal with the Co concentration within the synthesis solution. The line of the $0 \%$ is divided by 100 .

To explain the gradual decrease of the CDs single with the increase of $\mathrm{Co}$ in the synthesis solution, three main interpretations are possible: firstly, the presence of Co in the synthesis solution may directly inhibit the formation of this $\mathrm{CD}$ during the growth. Secondly, the paramagnetic incorporated $\mathrm{Co}^{2+}$ ions and $\mathrm{CD}$ may form a non-magnetic complex, which is then not detectable by the EPR, and finally, another type of non-magnetic 
incorporated Co may break down the $\mathrm{CD}$, for instance by filling a vacancy. It has also been proposed that the disappearance of the $\mathrm{g} \sim 1.96$ signal when the TMs ions are incorporated in $\mathrm{ZnO}$ can only be due to the significant broadening due to spin-spin relaxation with the $3 d$ electrons. However, it is difficult to imagine $\mathrm{Co}^{2+}$ ions interacting with a $\mathrm{CD}$ without being altered in its magnetic parameters so that, as only $\mathrm{Co}^{2+}$ ions with bulk parameters are detected, this proposal may be finally equivalent to the second explanation. ${ }^{[34]}$

The physics of $\mathrm{ZnO}$ intrinsic and extrinsic defects are so plentiful that many types of defects complexes are likely to take place. Regarding our samples, we first note that in all of them, except the $\mathrm{g} \sim 1.96 \mathrm{CD}$ signal, no other defect or pollution-related lines are detected, indicating to the high crystal quality and purity of the NRs under investigation. The CD signal detected on the pure $\mathrm{ZnO}$ NRs ( $0 \%$ Co)as shown in Figure 5.19 is made of at least two close lines, which collapse into a single asymmetric line for $\theta$ $=90^{\circ}$. Here, one of these observed lines appears to be almost isotropic $\left(\mathrm{g}_{\perp}\right.$ $\left.\cong \mathrm{g}_{\|}\right)$, while the other one displays a slight easy-axis anisotropy $\left(\mathrm{g}_{\perp} \neq \mathrm{g}_{\|}\right)$ signed by the displacement of the positive peak. This shift allows us to estimate $\Delta g\left(\Delta g=g_{\perp}-g_{\|}\right)$for the anisotropic line to be -0.0014 , thus likely to be related to a $\mathrm{Zn}_{\mathrm{i}}^{+}$defect $(\Delta \mathrm{g}=-0.0010)$ within the ordered phase of NRs. Regarding the isotropic line, although an isotropic defect cannot be excluded, it is likely to be related to the disordered phase present in the Co-doped samples. If so, the line may arise from a powder of the anisotropic defect, with slightly modified parameters: this could be the case for disordered Co-doped $\mathrm{ZnO}$ large nanoparticles or partially grown NRs. Consequently, at this stage, we cannot decide an exact answer to this question. In particular, because the precise origin of the CDs is not known with certainty. Further experimental and theoretical investigations have to 
be performed. For example, ab initio theoretical study of $\mathrm{ZnO}$ supercells containing different types of paramagnetic defects (compatible with slight easy axis magnetic anisotropy observed in this work) and substitutional $\mathrm{Co}^{2+}$ ions in various relative positions would give more insight into answering these questions. We show that only a little amount of $\mathrm{Co}$ in the synthesis solution (about $0.2 \%$ ) is necessary to suppress almost all these paramagnetic CDs. ${ }^{[34]}$ These results give insight in the experimentally observed improvement of the crystal quality of diluted Co-doped $\mathrm{ZnO}$ NRs, as well as into the control of paramagnetic defects in ZnO NRs.

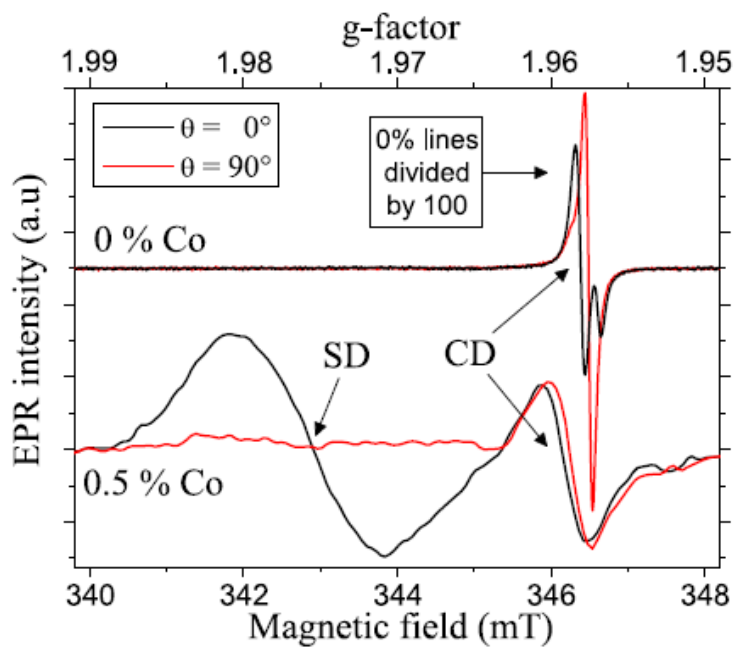

Figure 5.19: Anisotropy of the EPR CD signal in pure $(0 \% \mathrm{Co})$ and Codoped $(0.5 \%)$ samples, with respect to the magnetic field orientation, recorded at $T=6 \mathrm{~K}$. Sapphire defect $(S D)$ signal present in the $0.5 \%$ sample is shown for the $\theta=0^{\circ}$ orientation. 


\section{Research summary and future work}

Zinc oxide $(\mathrm{ZnO})$ nanorods $(\mathrm{NRs})$ exhibit unique optical and electronic properties, which are of particular interest in the development of optoelectronic devices e.g., ultraviolet (UV) photodetectors, light-emitting diodes (LEDs) and solar cells. In addition to this $\mathrm{ZnO}$ is a piezoelectric and biocompatible material. The potential of $\mathrm{ZnO} \mathrm{NRs}$ in all applications mentioned above, however, would require synthesis of high crystal quality with in-depth knowledge in understanding their optical and electronic properties. As the low-temperature aqueous chemical synthesis (LT-ACS) holds great promise for the synthesis of $\mathrm{ZnO}$ NRs and offers the possibility to tune their final properties by adjusting the synthesis parameters, intense research efforts are devoted in this respect. In this regard, vertically wellaligned $\mathrm{ZnO} \mathrm{NRs}$ with optimized point defects concentration and knowledge about their spatial distribution along the NRs were achieved. Also, high crystal quality well-oriented Co-doped $\mathrm{ZnO}$ NRs were obtained in this thesis. In paper I, by varying the stirring durations for the synthesis solutions, we were able to tune the defects emission along the NRs, and this is most likely attributed to the variation of $\mathrm{OH}^{-}$concentration during the growth process by either high or low super-saturations. Then, by choosing the best stirring duration regarding the vertical alignment and concentration of defects achieved in the paper I, combined with $\mathrm{ZnO}$ seed layer precursor's types, and its molar ratios are studied in papers II and III, and we tuned the concentration of interface defects at the synthesized n$\mathrm{ZnO} \mathrm{NRs} / \mathrm{p}-\mathrm{GaN}$ heterostructured interface. A significant improvement in the electroluminescence (EL) intensity was observed for the fabricated heterojunction LEDs based on the results of papers II and III. The improved EL intensity from the n-ZnO NRs-based heterojunction LEDs was likely attributed to reduced density of non-radiative related defect 
using an optimized molar ratio and a specific precursor type utilized for the $\mathrm{ZnO}$ seed layer. After that, in papers IV-VI, high crystal quality vertically well-oriented $\mathrm{ZnO}$ NRs has been demonstrated by incorporating diluted cobalt $(\mathrm{Co})$ inside the $\mathrm{ZnO}$ NRs crystal lattice. Finally, a concentration study (paper VI) of the synthesized vertically well-aligned pure and Co-doped $\mathrm{ZnO} \mathrm{NRs}$ at low-temperature suggested that a relatively small amount of $\mathrm{Co}^{2+}(0.2 \%)$ is sufficient to suppress almost all core-defects (CDs) that were observed in pure $\mathrm{ZnO}$ NRs. In view of the different results from all the complementary measurements on the synthesized pure and Co-doped $\mathrm{ZnO} \mathrm{NRs}$, we can summarize that: the substitution of the $\mathrm{Co}^{2+}$ inside the $\mathrm{ZnO}$ NRs supplies a highly anisotropic magnetic $\mathrm{Co}^{2+}$ signal defined by eight-lines suggesting that the synthesized NRs are single crystalline, vertically well-aligned, and at least the Co is homogeneously distributed along the NRs. Also, the substitution of the $\mathrm{Co}^{2+}$ was observed to be identified by a drastic reduction in the CDs signal ( $\mathrm{g}$ 1.956) seen in pure $\mathrm{ZnO}$ NRs. This decrease in the CDs signal is presumably due to the presence of Co in the synthesis solution may instantly hinder the formation of these CDs during the growth process. Another probability is that the paramagnetic incorporated $\mathrm{Co}^{2+}$ ions and $\mathrm{CD}$ may create a non-magnetic complex, which is not detectable by electron paramagnetic resonance (EPR), or a different type of nonmagnetic incorporated Co might break down the $\mathrm{CD}$, for example by filling a vacancy. Moreover, the EPR result indicates that the resolved anisotropy of the $\mathrm{CD}$ signal at $\mathrm{g} \sim 1.96$ in pure NRs highly proposes that $\mathrm{CD}$ arise, at least partially, from the $\mathrm{Zn}_{\mathrm{i}}^{+}$shallow donor from the core of the NRs rather than the shell.

Despite tremendous effort devoted to synthesizing high crystalline quality vertically well-oriented Co-doped $\mathrm{ZnO}$ NRs using the LT-ACS, 
there are many challenges to overcome. For instance, more experimental research work is needed in adjusting the synthesis parameters to improve the incorporated amount of the $\mathrm{Co}^{2+}$ ions into the $\mathrm{ZnO}$ NRs crystal lattice while keeping control over the crystal quality, morphology, and orientations. This step will probably lead to the possible observation of room-temperature ferromagnetism (RT-FM) from the LT-ACS synthesized Co-doped $\mathrm{ZnO}$ NRs. Further, advanced spectroscopy investigations e.g., photoluminescence excitation (PLE) and optically detected magnetic resonance (ODMR) performed at low-temperature and supported by ab initio theoretical study of $\mathrm{ZnO}$ super-cells containing various types of paramagnetic defects and substitutional $\mathrm{Co}^{2+}$ ions in different relative positions would give insights into the origin of the CDs. Furthermore, the potential of the synthesis of optimized Co-doped $\mathrm{ZnO}$ NRs for spintronic-based devices e.g., UV photodetectors, LEDs, and magnetic capacitors can be utilized. 


\section{References}

[1] H. S. Nalwa, Handbook of Nanostructured Materials and Nanotechnology, Academic Press, New York, (2000).

[2] C. N. R. Rao, A. Mueller, and A. K. Cheetham, The Chemistry of Nanomaterials: Synthesis, Properties, and Applications, Wiley-VCH, Weinheim, (2004).

[3] G. Cao and Y. Wang, Nanostructures and Nanomaterials: Synthesis, Properties and Applications, World Scientific Publishing Co. Pte. Ltd., Singapore (2011).

[4] O. I. Micic, C. J. Curtis, K. M. Jones, J. R. Sprague, and A. J. Nozik, Synthesis and Characterization of InP Quantum Dots, J. Phys. Chem. 98, 4966 (1994).

[5] T. S. Ahmadi, Z. L. Wang, T. C. Green, A. Henglein, and M. A. El-Sayed, Shape-Controlled Synthesis of Colloidal Platinum Nanoparticles, Science 272, 1924 (1996).

[6] Y. Sun, and Y. Xia, Shape-Controlled Synthesis of Gold and Silver Nanoparticles, Science 298, 2176 (2002).

[7] W. K. Bae, K. Char, H. Hur, and S. Lee, Single-Step Synthesis of Quantum Dots with Chemical Composition Gradients, Chem. Mater. 20, 531 (2008).

[8] C. Wang, H. Daimon, T. Onodera, T. Koda, and S. Sun, A General Approach to the Size and Shape-Controlled Synthesis of Platinum Nanoparticles and Their Catalytic Reduction of Oxygen, Angew. Chem. 47, 3588 (2008).

[9] L. Vayssieres, Growth of Arrayed Nanorods and Nanowires of ZnO from Aqueous Solutions, Adv. Mater. 15, 464 (2003).

[10] Y. Xia, P. Yang, Y. Sun, Y. Wu, B. Mayers, B. Gates, Y. Yin, F. Kim, and H. Yan, One-Dimensional Nanostructures: Synthesis, Characterization, and Applications, Adv. Mater. 15, 353 (2003).

[11] A. Gole, and C. J. Murphy, Seed-Mediated Synthesis of Gold Nanorods: Role of the Size and Nature of the Seed, Chem. Mater. 16, 3633 (2004).

[12] A. K. Geim, and K. S. Novoselov, The rise of graphene, Nat. Mater. 6, 183 (2007). 
[13] Y. Wu, P. Qiao, T. Chong, and Z. Shen, Carbon Nanowalls Grown by Microwave Plasma Enhanced Chemical Vapor Deposition, Adv. Mater. 14, 64 (2002).

[14] M.-S. Wu, Y.-H. Ou, and Y.-P. Lin, Iron Oxide Nanosheets and Nanoparticles Synthesized by a Facile Single-Step Coprecipitation Method for Lithium-Ion Batteries, Journal of The Electrochemical Society 158 (3), A231 (2011).

[15] B. Cao, W. Cai, Y. Li, F. Sun, and Z. L. Wang, Ultraviolet-light-emitting $\mathrm{ZnO}$ nanosheets prepared by a chemical bath deposition method, Nanotechnology 16, 1734 (2005).

[16] Z. R. Tian, J. A. Voigt, J. Liu, B. Mckenzie, M. J. Mcdermott, M. A. Rodriguez, $\mathrm{H}$. Konishi, and $\mathrm{H}$. $\mathrm{Xu}$, Complex and oriented $\mathrm{ZnO}$ nanostructures, Nat. Mater. 2, 821 (2003).

[17] Z. L. Wang, Nanostructures of zinc oxide, Mater.Today 7, 26 (2004).

[18] L. E. Greene, M. Law, D. H. Tan, M. Montano, J. Goldberger, G. Somorjai, and P. Yang, General Route to Vertical ZnO Nanowire Arrays Using Textured ZnO Seeds, Nano Lett. 7, 1231 (2005).

[19] S. Xu and Z. L. Wang, One-dimensional ZnO nanostructures: Solution growth and functional properties, Nano Res. 4, 1013 (2011).

[20] U. Özgür, Ya. I. Alivov, C. Liu, A. Teke, M. A. Reshchikov, S. Doğan, V. Avrutin, S.-J. Cho, and $\mathrm{H}$. Morkoç, A comprehensive review of $\mathrm{ZnO}$ materials and devices, J. Appl. Phys. 98, 041301 (2005).

[21] C. Klingshirn, ZnO: Material, Physics and Applications, Chem. Phys. Chem. 8, 782 (2007).

[22] M. Willander et al., Zinc oxide nanorod based photonic devices: recent progress in growth, light emitting diodes and lasers, Nanotechnology 20, 332001 (2009).

[23] A. B. Djurišić, A. M. C. Ng, and X. Y. Chen, ZnO nanostructures for optoelectronics: Material properties and device applications, Prog. Quantum Electron. 34, 191 (2010).

[24] Y. Jin, J. Wang, B. Sun, J. C. Blakesley, and N. C. Greenham, SolutionProcessed Ultraviolet Photodetectors Based on Colloidal ZnO Nanoparticles, Nano Lett. 8 (6), 1649 (2008). 
[25] C. Soci, A. Zhang, B. Xiang, S. A. Dayeh, D. P. R. Aplin, J. Park, X. Y. Bao, Y. H. Lo, and D. Wang, ZnO Nanowire UV Photodetectors with High Internal Gain, Nano Lett. 4, 103 (2007).

[26] L. W. Ji, S. M. Peng, Y. K. Su, S. J. Young, C. Z. Wu, and W. B. Cheng, ultraviolet photodetectors based on selectively grown $\mathrm{ZnO}$ nanostructures, Appl. Phys. Lett. 94, 203106 (2009).

[27] C. O. Chey, X. Liu, H. Alnoor, O. Nur, and M. Willander, Fast piezoresistive sensor and UV photodetector based on Mn-doped $\mathrm{ZnO}$ nanorods, Phys. Status Solidi RRL 9, 87 (2015).

[28] M. Law, L. E. Greene, J. C. Johnson, R. Saykally, and P. Yang, Nanowire dye-sensitized solar cells, Nat. Mater. 4, 455 (2005).

[29] C.-W. Liu, S.-J. Chang, S. Brahma, C.-H. Hsiao, F. M. Chang, P. H. Wang, and K. Y. Lo, Hydrothermally grown oriented $\mathrm{ZnO}$ nanorod arrays for gas sensing applications, J. Appl. Phys. 117, 084315 (2015).

[30] F. A. Kohan, G. Ceder, D. Morgan, and C. G. Van De Walle, Firstprinciples study of native point defects in ZnO, Phys. Rev. B 61, 15019 (2000)

[31] C. G. Van De Walle, Defect analysis and engineering in ZnO, Physica B 308-310, 899 (2001).

[32] D. Li, Y. H. Leung, A. B. Djurišić, Z. T. Liu, M. H. Xie, S. L. Shi, S. J. Xu, and W. K. Chan, Different origins of visible luminescence in $\mathrm{ZnO}$ nanostructures fabricated by the chemical and evaporation methods, Appl. Phys. Lett. 85, 1601 (2004).

[33] K. H. Tam, C. K. Cheung, Y. H. Leung, A. B. Djurišić, C. C. Ling, C. D. Beling, S. Fung, W. M. Kwok, W. K. Chan, D. L. Phillips, L. Ding, and W. K. $\mathrm{Ge}$, Defects in ZnO Nanorods Prepared by a Hydrothermal Method, J. Phys. Chem. B 110, 20865 (2006).

[34] A. Savoyant, H. Alnoor, O. Pilone, O. Nur, and M. Willander, Coredefect reduction in $\mathrm{ZnO}$ nanorods by cobalt incorporation, Nanotechnology 28, 285705 (2017).

[35] A. Zainelabdin, S. Zaman, G. Amin, O. Nur, and M. Willander, Deposition of Well-Aligned ZnO Nanorods at $50{ }^{\circ} \mathrm{C}$ on Metal, Semiconducting Polymer, and Copper Oxides Substrates and Their Structural and Optical Properties, Cryst. Growth Des. 10, 3250 (2010). 
[36] M. H. Asif, S. M. U. Ali, O. Nur, M. Willander, C. Brännmark, P. Strålfors, U. H. Englund, F. Elinder, and B. Danielsson, Functionalised ZnO-nanorodbased selective electrochemical sensor for intracellular glucose, Biosensors and Bioelectronics 25, 2205 (2010).

[37] Z. L. Wang and S. J, Piezoelectric nanogenerators based on zinc oxide nanowire arrays, Science 312, 242 (2006).

[38] A. Kumar, H. Gullapalli, K. Balakrishnan, A. Botello-Mendez, R. Vajtai, M. Terrones, and P. M. Ajayan, Flexible ZnO-Cellulose Nanocomposite for Multisource Energy Conversion, Small 7, 2173 (2011).

[39] L. E. Greene, B. D. Yuhas, M. Law, D. Zitoun, and P. Yang, SolutionGrown Zinc Oxide Nanowires, Inorganic Chemistry 19, 7535 (2006).

[40] L. E. Greene, M. Law, J. Goldberger, F. Kim, J. C. Johnson, Y. Zhang, R. J. Saykally, and P. Yang, Low-Temperature Wafer-Scale Production of ZnO Nanowire Arrays, Angew, Chem. Int.Ed. 42, 3031 (2003).

[41] E. De la Rosa, S. Sepúlveda-Guzman, B. Reeja-Jayan, A. Torres, P. Salas, N. Elizondo, and M. Jose Yacaman, Controlling the Growth and Luminescence Properties of Well-Faceted ZnO Nanorods, J. Phys. Chem. C 111, 8489 (2007).

[42] A. Escobedo-Morales and U. Pal, Defect annihilation and morphological improvement of hydrothermally grown $\mathrm{ZnO}$ nanorods by $\mathrm{Ga}$ doping, Appl. Phys. Lett. 93, 193120 (2008).

[43] A. B. Djurišić, Y. H. Leung, K. H. Tam, Y. F. Hsu, L. Ding, W. K. Ge, Y. C. Zhong, K. S. Wong, W. K. Chan, H. L. Tam, K.W. Cheah, W. M. Kwok, and D. L. Phillips, Defect emissions in ZnO nanostructures, Nanotechnology 18, 095702 (2007).

[44] A. Zainelabdin, S. Zaman, G. Amin, O. Nur, and M. Willander, Stable White Light Electroluminescence from Highly Flexible Polymer/ZnO Nanorods Hybrid Heterojunction Grown at $50^{\circ} \mathrm{C}$, Nanoscale Res Lett. 5, 1442 (2010).

[45] T. M. Børseth, B. G. Svensson, A. Y. Kuznetsov, P. Klason, Q. X. Zhao, and $\mathrm{M}$. Willander, Identification of oxygen and zinc vacancy optical signals in ZnO, Appl. Phys. Lett. 89, 262112 (2006). 
[46] N. H. Alvi, K. ul Hasan, O. Nur, and M. Willander, The origin of the red emission in $n-Z n O$ nanotubes/p-GaN white light emitting diodes, Nanoscale Res. Lett. 6, 130 (2011).

[47] J. K. Furdyna, Diluted magnetic semiconductors, J. Appl. Phys. 4, 15 (1988).

[48] K. R. Kittilstved, W. K. Liu, and D. R. Gamelin, Electronic structure origins of polarity-dependent high-TC ferromagnetism in oxide-diluted magnetic semiconductors, Nat. Mater. 5, 291 (2006).

[49] P. Sharma, A. Gupta, K. V. Rao, F. J. Owens, R. Sharma, R. Ahuja, J. M. Osorio Guillen, B. Johansson, and G. A. Gehring, Ferromagnetism above room temperature in bulk and transparent thin films of $\mathrm{Mn}$-doped $\mathrm{ZnO}$, Nat. Mater. 2, 673 (2003).

[50] Z. H. Zhang, X. Wang, J. B. Xu, S. Muller, C. Ronning, and Q. Li, Evidence of intrinsic ferromagnetism in individual dilute magnetic semiconducting nanostructures, Nat. Nanotechnology. 4, 523 (2009).

[51] P. Lommens, F. Loncke, P. F. Smet, F. Callens, D. Poelman, H. Vrielinck, and Z. Hens, Dopant Incorporation in Colloidal Quantum Dots: A Case Study on $\mathrm{Co}^{2+}$ Doped $\mathrm{ZnO}$, Chem. Mater. 19, 5576 (2007).

[52] A. Savoyant, H. Alnoor, S. Bertaina, O. Nur, and M. Willander, EPR investigation of pure and Co-doped $\mathrm{ZnO}$ oriented nanocrystals, Nanotechnology 28, 035705 (2017).

[53] K. Govender, S. D. Boyle, B. P. Kenway, and P. O'Brien, Understanding the factors that govern the deposition and morphology of thin films of $\mathrm{ZnO}$ from aqueous solution, J. Mater. Chem 14, 2575 (2004).

[54] S. Xu, C. Lao, B. Weintraub, and Z. L. Wang, Density-controlled growth of aligned $\mathrm{ZnO}$ nanowire arrays by seedless chemical approach on smooth surfaces, J. Mater. Res. 23, 2072 (2008).

[55] Y. Zhang, M. K. Ram, E. K. Stefanakos, and D. Y. Goswami, Synthesis, Characterization, and Applications of ZnO Nanowires, J. Nanomater. 2012, 1 (2012).

[56] H. Alnoor, C. O. Chey, G. Pozina, X. Liu, V. Khranovskyy, M. Willander, and $\mathrm{O}$. Nur, Effect of precursor solutions stirring on deep level defects concentration and spatial distribution in low temperature aqueous chemical synthesis of zinc oxide nanorods, AIP Advances 5, 087180 (2015). 
[57] H. Alnoor, G. Pozina, V. Khranovskyy, X. Liu, D. landolo, M. Willander, and $\mathrm{O}$. Nur, Influence of $\mathrm{ZnO}$ seed layer precursor molar ratio on the density of interface defects in low temperature aqueous chemically synthesized ZnO nanorods/GaN light-emitting diodes, J. Appl. Phys. 119, 165702 (2016).

[58] H. Alnoor, G. Pozina, M. Willander, and O. Nur, Seed layer synthesis effect on the concentration of interface defects and emission spectra of $\mathrm{ZnO}$ nanorods/p-GaN light-emitting diode, Phys. Status Solidi A 214, 1862 (2017).

[59] H. Alnoor, A. Savoyant, X. Liu, G. Pozina, M. Willander, and O. Nur, An effective low-temperature solution synthesis of Co-doped [0001]-oriented ZnO nanorods, J. Appl. Phy. 121, 215102 (2017).

[60] J. Song and S. Lim, Effect of Seed Layer on the Growth of ZnO Nanorods, J. Phys. Chem. C 111, 596 (2007).

[61] L.-W. Ji, S.-M. Peng, J.-S. Wu, W.-S. Shih, C.-Z. Wu, and I-T. Tang, Effect of seed layer on the growth of well-aligned $\mathrm{ZnO}$ nanowires, Journal of Physics and Chemistry of Solids 70, 1359 (2009).

[62] S.-W. Chen and J.-M. Wu, Nucleation mechanisms and their influences on characteristics of $\mathrm{ZnO}$ nanorod arrays prepared by a hydrothermal method, Acta Mater. 59, 841 (2011).

[63] A. M. C. Ng, X. Y. Chen, F. Fang, Y. F. Hsu, A. B. Djurišić, C. C. Ling, H. L. Tam, K. W. Cheah, P. W. K. Fong, H. F. Lui, C. Surya, and W. K. Chan, Solution-based growth of ZnO nanorods for light-emitting devices: hydrothermal vs. electrodeposition, Appl. Phys. B 100, 851 (2010).

[64] A. M. C. Ng, Y. Y. Xi, Y. F. Hsu, A. B. Djurišić, W. K. Chan, S. Gwo, H. L. Tam, K. W. Cheah, P. W. K. Fong, H. F. Lui, and C. Surya, GaN/ZnO nanorod light emitting diodes with different emission spectra, Nanotechnology 20, 445201 (2009).

[65] X. Y. Chen, A. M. C. Ng, F. Fang, A. B. Djurišić, W. K. Chan, H. L. Tam, K. W. Cheah, P. W. K. Fong, H. F. Lui, and C. Surya, The Influence of the ZnO Seed Layer on the ZnO Nanorod/GaN LEDs, J. Electrochem. Soc. 157 (3), H308 (2010). 
[66] W. Zhou, R. Apkarian, Z. L. Wang, and D. Joy, Fundamentals of Scanning Electron Microscopy (SEM): Scanning Microscopy for Nanotechnology, Springer New York, 1-40 (2007).

[67] G. Will, Powder Diffraction, Springer Berlin Heidelberg, 1-39 (2006).

[68] J. F. Moulder, W. F. Stickle, P.E.'Sobol, and K. D. Bomben, Handbook of $X$-ray Photoelectron Spectroscopy, Physical Electronics USA, Inc., (1995).

[69] H.-L. Lee and N. T. Flynn, Handbook of Applied Solid State Spectroscopy, X-RAY PHOTOELECTRON SPECTROSCOPY, 485 (2006).

[70] G. D. Gilliland, photoluminescence spectroscopy of crystalline semiconductors, Mater. Sci. Eng., R. 18, 99 (1997).

[71] B. G. Yacobi and D.B. Holt, Cathodoluminescence Microscopy of inorganic solids, Plenum Press, New York (1990).

[72] A. Gustafsson, M.-E. Pistol, L. Montelius, and L. Samuelson, Local probe techniques for luminescence studies of low-dimensional semiconductor structures, J. Appl. Phys. 84, 1715 (1998).

[73] L. J. Brillson, Applications of depth-resolved cathodoluminescence spectroscopy, J. Phys. D: Appl. Phys., 45, 183001 (2012).

[74] K. Thonke, I. Tischer, M. Hocker, M. Schirra, K. Fujan, M. Wiedenmann, R. Schneider, M. Frey, and M. Feneberg, Nanoscale characterization of semiconductors by cathodoluminescence spectroscopy, IOP Conf. Series: Mater. Sci. Eng., 55, 012018 (2014).

[75] L. J. Brillson, W. T. Ruane, H. Gao, Y. Zhang, J. Luo, H. von Wenckstern, and M. Grundmann, Spatially-resolved cathodoluminescence spectroscopy of ZnO defects, Mater. Sci. Semicond. Process. 57, 197 (2017).

[76] MonoCL4 Cathodoluminescence System (SEM-CL) Gatan, Inc.

[77] A. B. Djurišić, W. C. H. Choy, V. A. L. Roy, Y. H. Leung, C. Y. Kwong, K. W. Cheah, T. K. Gundu Rao, W. K. Chan, H. Fei Lui, and C. Surya, Photoluminescence and Electron Paramagnetic Resonance of $\mathrm{ZnO}$ Tetrapod Structures, Adv. Funct. Mater. 14, 856 (2004).

[78] B. P. Renee, L. F. Clark, and A. G. Brian, Epitaxial Chemical Deposition of ZnO Nanocolumns from NaOH Solutions, Langmuir 20, 5114 (2004). 
[79] S.-F. Wang, T.-Y. Tseng, Y.-R. Wang, C.-Y. Wang, H.-C. Lu, and W.-L. Shih, Effects of Preparation Conditions on the Growth of ZnO Nanorod Arrays Using Aqueous Solution Method, Int. J. Appl. Ceram. Technol. 5, 419 (2008).

[80] W. I. Park and G. C. Yi, Electroluminescence in n-ZnO Nanorod Arrays Vertically Grown on p-GaN, Adv. Mater. 16, 87 (2004).

[81] O. Lupan, T. Pauporté, B. Viana, I. M. Tiginyanu, V. V. Ursaki, and R. Cortés, Epitaxial Electrodeposition of ZnO Nanowire Arrays on $p$-GaN for Efficient UV-Light-Emitting Diode Fabrication, ACS Appl. Mater. Interfaces 2, 2083 (2010).

[82] A. Echresh, C. O. Chey, M. Z. Shoushtari, O. Nur, and M. Willander, Tuning the emission of $\mathrm{ZnO}$ nanorods based light emitting diodes using $\mathrm{Ag}$ doping, J. Appl. Phys. 116, 193104 (2014).

[83] X. Chen, A. M. C. Ng, F. Fang, Y. H. Ng, A. B. Djurišić, H. L. Tam, K.W. Cheah, S. Gwo, W. K. Chan, P. W. K. Fong, H. F. Lui, and C. Surya, ZnO nanorod/GaN light-emitting diodes: The origin of yellow and violet emission bands under reverse and forward bias, J. Appl. Phys. 110, 094513 (2011).

[84] Z. Shi, Y. T. Zhang, X. J. Cui, B. Wu, S. W. Zhuang, F. Yang, X. T. Yang, B. L. Zhang, and G. T. Zhang, Improvement of electroluminescence performance by integration of $\mathrm{ZnO}$ nanowires and single-crystalline films on ZnO/GaN heterojunction, Appl. Phys. Lett. 104, 131109 (2014).

[85] S. Xu, C. Xu, Y. Liu, Y. F. Hu, R. Yang, Q. Yang, J. H. Ryou, H. J. Kim, Z. Lochner, S. Choi, R. Dupuis, and Z. L. Wang, Ordered Nanowire Array Blue/Near-UV Light Emitting Diodes, Adv. Mater. 22, 4749 (2010).

[86] H. Y. Lee, C. T. Lee, and J. T. Yan, Emission mechanisms of passivated single $n$-ZnO: In/i-ZnO/p-GaN-heterostructured nanorod light-emitting diodes, Appl. Phys. Lett. 97, 111111 (2010).

[87] Z. Shi, Y. T. Zhang, X. J. Cui, B. Wu, S. W. Zhuang, F. Yang, X. T. Yang, B. L. Zhang, and G. T. Zhang, Improvement of electroluminescence performance by integration of $\mathrm{ZnO}$ nanowires and single-crystalline films on ZnO/GaN heterojunction, Appl. Phys. Lett. 104, 131109 (2014). 
[88] S. Anantachaisilp, S. M. Smith, C. Ton-That, T. Osotchan, R. A. Moon, and R. M. Phillips, Tailoring Deep Level Surface Defects in ZnO Nanorods for High Sensitivity Ammonia Gas Sensing, J.Phys. Chem. C 118, 27150 (2014).

[89] A. Simimol, N. T. Manikandanath, A. A. Anappara, P. Chowdhury, and C. H. Barshilia, Tuning of deep level emission in highly oriented electrodeposited $\mathrm{ZnO}$ nanorods by post growth annealing treatments, J. Appl. Phys. 116, 074309 (2014).

[90] O. Lupan, T. Pauporte, B. Viana, V. V. Ursaki, I. M. Tiginyanu, V.Sontea, and L. Chow, UV-Blue and Green Electroluminescence from Cu-Doped ZnO Nanorod Emitters Hydrothermally Synthesized on p-GaN, J. Nanoelectron. Optoelectron. 7, 712 (2012).

[91] H. H. Huang, G. J. Fang, Y. Li, S. Z. Li, X. M. Mo, H. Long, H. N. Wang, D. L. Carroll, and X. Z. Zhao, Improved and color tunable electroluminescence from $n-\mathrm{ZnO} / \mathrm{HfO} / \mathrm{p}$-GaN heterojunction light emitting diodes, Appl. Phys. Lett. 100, 233502 (2012).

[92] G. C. Park, S. M. Hwang, S. M. Lee, J. H. Choi, K. M. Song, H. Y. Kim, H.S. Kim, S.-J. Eum, S.-B. Jung, J. H. Lim, and J. Joo, Hydrothermally Grown Indoped ZnO Nanorods on p-GaN Films for Color-tunable Heterojunction Light-emitting-diodes, Sci. Rep. 5, 10410 (2015).

[93] S. G. Zhang, X. W. Zhang, J. X. Wang, J. B. You, Z. G. Yin, J. J. Dong, B. Cui, A. M. Wowchak, A. M. Dabiran, and P. P. Chow, Enhanced electroluminescence from $\mathrm{ZnO}$-based heterojunction light-emitting diodes by hydrogen plasma treatment, Phys. Status Solidi RRL 5, 74 (2011).

[94] J. J. Dong, X. W. Zhang, Z. G. Yin, J. X. Wang, S. G. Zhang, F. T. Si, H. L. Gao, and X. Liu, Ultraviolet electroluminescence from ordered $\mathrm{ZnO}$ nanorod array/p-GaN light emitting diodes, Appl. Phys. Lett. 100, 171109 (2012).

[95] J. Cui, Q. Zeng, and U. J. Gibson, Synthesis and magnetic properties of Co-doped ZnO nanowires, J. Appl. Phys. 99, 08M113 (2006).

[96] B. Panigrahy, M. Aslam, and D. Bahadur, Aqueous Synthesis of Mn- and Co-Doped ZnO Nanorods, J. Phys. Chem. C 114, 11758 (2010).

[97] S. Shi, Y. Yang, J. Xu, L. Li, X. Zhang, G.-H. Hu, and Z.-M. Dang, Structural, optical and magnetic properties of Co-doped ZnO nanorods prepared by hydrothermal method, Journal of Alloys and Compounds 576, 59 (2013). 
[98] A. Simimol, A. A. Anappara, S. Greulich-Weber, P. Chowdhury, and H. C. Barshilia, Enhanced room temperature ferromagnetism in electrodeposited Co-doped $\mathrm{ZnO}$ nanostructured thin films by controlling the oxygen vacancy defects, J. Appl. Phys. 117, 214310 (2015).

[99] P. H. Kasai, Electron Spin Resonance Studies of Donors and Acceptors in ZnO, Phys. Rev. 130, 989 (1963).

[100] P. Jakes and E. Erdem, Finite size effects in ZnO nanoparticles: An electron paramagnetic resonance (EPR) analysis, Phys. Status Solidi RRL 5, 56 (2011).

[101] H. Kaftelen, K. Ocakoglu, R. Thomann, S. Tu, S. Weber, and E. Erdem, EPR and photoluminescence spectroscopy studies on the defect structure of ZnO nanocrystals, Phys. Rev. B 86, 014113 (2012).

[102] D. Sett and D. Basak, Highly enhanced $\mathrm{H}_{2}$ gas sensing characteristics of Co: ZnO nanorods and its mechanism, Sens. Actuators, B 243, 475 (2017).

[103] M. H. Chu, G. Marínez-Criado, J. Segura-Ruiz, S. Geburt, and C. Ronning, Nano-X-ray diffraction study of single Co-implanted $\mathrm{ZnO}$ nanowires, Phys. Status Solidi A 211, 2523 (2014).

[104] J. H. Park, Y. J. Lee, J. S. Bae, B.-S. Kim, Y. C. Cho, C. Moriyoshi, Y. Kuroiwa, S. Lee, and S.-Y. Jeong, Analysis of oxygen vacancy in Co-doped $\mathrm{ZnO}$ using the electron density distribution obtained using MEM, Nanoscale Res. Lett. 10, 186 (2015).

[105] C.-W. Liu, S.-J. Chang, S. Brahma, C.-H. Hsiao, F. M. Chang, P. H. Wang, and K. Y. Lo, Enhancement in the structure quality of $\mathrm{ZnO}$ nanorods by diluted Co dopants: Analyses via optical second harmonic generation, J. Appl. Phys. 117, 084315 (2015).

[106] E. G. Barbagiovanni, R. Reitano, G. Franzó, V. Strano, A. Terrasi, and S. Mirabella, Radiative mechanism and surface modification of four visible deep level defect states in ZnO nanorods, Nanoscale 8, 995 (2016).

[107] W. Baiqi, S. Xudong, F. Qiang, J. Iqbal, L. Yan, F. Honggang, and Y. Dapeng, Photoluminescence properties of Co-doped $\mathrm{ZnO}$ nanorods array fabricated by the solution method, Physica E 41, 413 (2009). 
[108] S. K. S. Parashar, B. S. Murty, S. Repp, S. Weber, and E. Erdem, Investigation of intrinsic defects in core-shell structured $\mathrm{ZnO}$ nanocrystals, J. Appl. Phys. 111, 113712 (2012). 


\section{Papers}

The papers associated with this thesis have been removed for copyright reasons. For more details about these see:

http:// urn.kb.se/ resolve?urn=urn:nbn:se:liu:diva- 141753 